\title{
Targeted nanoagents for the detection of cancers
}

\section{Citation}

McCarthy, Jason R., Jayeeta Bhaumik, Mark R. Karver, S. Sibel Erdem, and Ralph Weissleder. 2010. "Targeted Nanoagents for the Detection of Cancers." Molecular Oncology 4 (6): 511-28. https://doi.org/10.1016/j.molonc.2010.08.003.

\section{Permanent link}

http://nrs.harvard.edu/urn-3:HUL.InstRepos:41384209

\section{Terms of Use}

This article was downloaded from Harvard University's DASH repository, and is made available under the terms and conditions applicable to Other Posted Material, as set forth at http:// nrs.harvard.edu/urn-3:HUL.InstRepos:dash.current.terms-of-use\#LAA

\section{Share Your Story}

The Harvard community has made this article openly available.

Please share how this access benefits you. Submit a story.

\section{Accessibility}




\title{
Review
}

\section{Targeted nanoagents for the detection of cancers}

\author{
Jason R. McCarthy*, Jayeeta Bhaumik, Mark R. Karver, S. Sibel Erdem, Ralph Weissleder
}

Center for Systems Biology, Harvard Medical School and Massachusetts General Hospital, 185 Cambridge Street, Suite 5.210, Boston, MA 02114, USA

A R T I C L E I N F O

Article history:

Received 12 April 2010

Received in revised form

17 August 2010

Accepted 17 August 2010

Available online 8 September 2010

Keywords:

Nanomedicine

Imaging

Affinity ligands

\begin{abstract}
A B S T R A C T
Nanotechnology has enabled a renaissance in the diagnosis of cancers. This is due, in part to the ability to develop agents bearing multiple functionalities, including those utilized for targeting, imaging, and therapy, allowing for the tailoring of the properties of the nanomaterials. Whereas many nanomaterials exhibit localization to diseased tissues via intrinsic targeting, the addition of targeting ligands, such as antibodies, peptides, aptamers, and small molecules, facilitates far more sensitive cancer detection. As such, this review focuses upon some of the most poignant examples of the utility of affinity ligand targeted nanoagents in the detection of cancer.
\end{abstract}

(c) 2010 Federation of European Biochemical Societies. Published by Elsevier B.V. All rights reserved.

\section{Introduction}

With the advent of nanotechnology, scientists have been given additional tools for the development of advanced materials for the diagnosis and treatment of disease. The usefulness of nanomaterials is derivative of a number of factors, including their small size, large surface area, and altered in vivo kinetics, as compared to small molecule-based agents. These properties also give rise to the creation of agents that are multifunctional, as nanoparticles are capable of being modified with a number of ligands, including those used to affect targeting, to image localization, or to deliver therapeutic molecules. The ability to synthesize multimodal nanoagents, or those bearing more than one type of imaging component, is also highly advantageous, as it allows for the maximal amount of data to be acquired from a single nanoagent preparation.
The application of nanomedicine to the diagnosis and treatment of cancer has been ongoing for over 20 years, although its clinical utility has yet to be fully realized (Retel et al., 2009). For example, superparamagnetic iron oxide nanoparticles, which have proven to be highly useful contrast agents for magnetic resonance imaging, have been utilized to increase the accuracy of cancer nodal staging (Ferrari, 2005; Harisinghani et al., 2003; Harisinghani and Weissleder, 2004), better delineate primary tumors (Enochs et al., 1999), image angiogenesis (Tang et al., 2005), and detect metastases (Harisinghani et al., 2001; Saini et al., 2000). While these initial agents relied upon the intrinsic ability of the materials to localize to diseased tissues, subsequent iterations have allowed for their targeting to sites of interest using a variety of ligands, including antibodies, peptides, aptamers, and small molecules. Herein, this review will focus upon the creation of targeted nanoagents for the improved detection of cancers.

\footnotetext{
* Corresponding author. Tel.: +1 617726 9218; fax: +1 6177265708.

E-mail address: jason_mccarthy@hms.harvard.edu (J.R. McCarthy).

1574-7891/\$ - see front matter @ 2010 Federation of European Biochemical Societies. Published by Elsevier B.V. All rights reserved. doi:10.1016/j.molonc.2010.08.003
} 
Given the breadth of this subject, this is not meant to be allencompassing. Instead, we have chosen some of the most prominent examples of targeted nanomaterials utilized for the imaging of cancers. Importantly, this review will discuss the methodologies utilized for the discovery of potential targeting ligands, and examine the ultimate utility of the resulting targeted nanoagents. For a more general overview of biomedical imaging in cancer detection and therapy please see (Fass, 2008).

\section{Nanomaterials in cancer imaging}

\subsection{Materials and modalities}

\subsubsection{Magnetic resonance imaging}

Magnetic resonance imaging (MRI) is a non-invasive technique that involves the disturbance of aligned nuclear spins in a strong magnetic field by a radio frequency pulse and the measurement of the realignment time of the nuclei to the magnetic field following termination of the pulse (Hornak, 2010). The realignment or relaxation time is tissue dependent allowing for the different magnetic gradients to be spatially localized to create an image with high tissue contrast based on differences in spin density (Kherlopian et al., 2008). Two types of relaxation times $\mathrm{T}_{1}$, or spin-lattice (longitudinal) and $\mathrm{T}_{2}$, or spin-spin (transverse), determine the signal for a particular tissue (Lin et al., 2009). $T_{1}$-weighted images are thus the result of longitudinal relaxation time and $\mathrm{T}_{2}$-weighted images rely on the rate of transverse relaxation to afford positive and negative contrast enhancement in the MR image respectively (Lin et al., 2009). MRI has become a valuable commodity in cancer detection due to its high soft tissue contrast, spatial resolution and penetration depth.

A significant amount of research in the field of medical MRI has focused on the development of contrast agents to improve image signal intensity, which can be low in their absence and is an inherent limitation of MRI. A majority of contrast agent research has focused on gadolinium (Gd)-based MRI agents due to this lanthanide's ability to decrease the $\mathrm{T}_{1}$ relaxation time, thus increasing the MR signal (Caravan et al., 1999). Unfortunately, one potential drawback of using paramagnetic Gd chelates is that relatively high concentrations are needed to achieve a sufficient increase in contrast signal resulting in a greater chance of acute toxicity (Caravan et al., 1999; Lin et al., 2009). In order to circumvent this, nanoparticulate strategies have been developed to increase the amount of complex delivered to a site of interest, such as tumors. For example, liposomal or micellar encapsulation of gadolinium chelates have successfully demonstrated the delivery of large payloads of these contrast agents to cancerous tissues (Mulder et al., 2005; Zhang et al., 2009).

In addition to contrast agent delivery vehicles, nanoparticles such as those composed of crystalline iron oxide, have themselves been utilized as negative contrast enhancers for MRI due to their ability to shorten T2 relaxation times. This property was first realized in the mid 1980s while imaging patients with hepatic iron overload (Stark et al., 1985). Studies shortly following this discovery demonstrated that small injectable ferrite particles were capable of detecting different types of cancer such as liver, splenic, and hepatic lymphoma (Saini et al., 1987; Weissleder et al., 1987a,b). More recent examples of using these superparamagnetic nanoparticles for the MRI detection of cancer include the visualization of otherwise evasive lymph node metastases in patients with prostate cancer (Harisinghani et al., 2003), as well as targeted nanoparticle conjugates that provided negative contrast enhancement for pancreatic cancer imaging (Montet et al., 2006). Further examples of the utility of iron oxide nanoparticles, along with several other nanomaterial-based strategies that have been utilized for MRI detection of cancer, have been recently reviewed (McCarthy et al., 2007; McCarthy and Weissleder, 2008; Lin et al., 2009).

\subsubsection{Nuclear imaging}

Positron emission tomography (PET) and single photon emission computed tomography (SPECT) are the two main modes of nuclear imaging. The major difference is that PET uses positron-emitting radionuclides such as ${ }^{18} \mathrm{~F},{ }^{11} \mathrm{C}$, and ${ }^{64} \mathrm{Cu}$, whereas SPECT uses radionuclides like ${ }^{111} \mathrm{In},{ }^{123} \mathrm{I}$, and ${ }^{99 \mathrm{~m}} \mathrm{TC}$ that emit gamma rays (Hamoudeh et al., 2008; Ting et al., 2009). PET scanners detect photons that are emitted $180^{\circ}$ from each other after collision of the emitted positron with a nearby electron. SPECT scanners, on the other hand, detect the single gamma rays emitted by a radionuclide (Hamoudeh et al., 2008). PET nuclides result in higher energy emission, which yields enhanced resolution, as compared to the gamma emission of SPECT nuclides, whereas SPECT nuclides possess longer half lives (Hamoudeh et al., 2008; Ting et al., 2009). The high sensitivity of detecting radionuclide emission is one of the main advantages PET and SPECT have over other imaging techniques.

A unique feature of nuclear imaging is the ability to image biological processes of cells and organs in their natural state, such as their metabolic activity (Kherlopian et al., 2008; Maurer, 2008). This, coupled with an increase in metabolism of cancerous cells, (Warburg, 1956) has allowed for the routine use of PET for tumor imaging using 2-[ $\left[{ }^{18} \mathrm{~F}\right]$ fluoro-2-deoxy-Dglucose (FDG) (Couturier et al., 2004). Many other ${ }^{18} \mathrm{~F}$-based molecules that rely on metabolism, such as radionuclidemodified amino acids, nucleosides, lipids, and hormones such as progesterone and androgen, have been developed and recently reviewed for the imaging of tumors (Couturier et al., 2004). Many examples are available for imaging tumors with SPECT as well, with the most commonly utilized radionuclides being ${ }^{99 \mathrm{~m}} \mathrm{Tc}$ and ${ }^{111} \mathrm{In}$, which have been routinely conjugated to antibodies or affinity ligands (Hamoudeh et al., 2008; Maurer, 2008; Mardirossian et al., 1996). Despite the high sensitivity of this modality, there are a number of concerns related to the potential toxicity of radiopharmaceuticals, which results in the limiting of the amount of radionuclide that can be safely administered. Therefore, a number of nanoparticulate systems have been investigated to provide a means of delivering concentrated amounts of the radiolabeled compound to the target site.

Studies reporting the use of liposomes for the delivery of radionuclides to tumorous tissue were reported over 30 years ago (Hamoudeh et al., 2008). Liposomes can be radiolabeled in several different ways, including encapsulation within the 
aqueous core, incorporation into the lipid bilayer, or surface modification, and have been successful in delivering radionuclides selectively to patients with head, neck, breast, and lung cancers (Harrington et al., 2001; Phillips, 1999). Many other strategies have been utilized to improve tumor-selective delivery of radionuclides such as polymeric micelles, hydrogels, and other nanoparticle formulations, all of which have been recently reviewed (Hamoudeh et al., 2008). PET or SPECT however, still suffer from a lack of spatial resolution, which is often resolved by combining these techniques with $\mathrm{x}$-ray computed tomography to afford anatomic localization (Maurer, 2008). Newer nanoparticle-based strategies combine different imaging functionalities on a single nanoparticle (Devaraj et al., 2009). The high sensitivity of nuclear imaging alone is a powerful tumor imaging technology, but coupled with other modalities, its usefulness is ever-improving.

\subsubsection{Fluorescence imaging}

Fluorescence imaging involves the detection of light emitted from molecules or materials subsequent to their excitation by light of a specific wavelength. The two most commonly utilized fluorescence imaging types for macroscopic intravital imaging are fluorescence reflectance imaging (FRI) and fluorescence molecular tomography (FMT), where the main difference is that the latter can provide three-dimensional quantitative information (Figure 1D), which is limited in the former (Figure 1C) (Graves et al., 2004). FRI can utilize a number of excitation sources, such as lasers, or broad spectrum lamps and filter sets, and detect planar images of emitted light using a charged coupled device (Figure 1A), whereas FMT systems utilize multiple excitation sources and emission detectors to produce a tomographic reconstruction of the image in three dimensions (Figure 1C) (Graves et al., 2004). The relative low cost and ability to simultaneously utilize multiple fluorophores have resulted in a considerable increase in the amount of fluorescent imaging research over the past decade.

Fluorescence imaging of cancerous tissue can be traced back to the mid 1900's where fluorescein was used to improve the detection of brain tumors (Moore et al., 1948). More recently, the discovery that near-infrared (NIR) photons are capable of deeper penetration into heterogeneous biological samples (Jobsis, 1977) has helped to improve upon the limitations of fluorescent imaging and led to an increase in biological applications for NIR fluorophores (Hilderbrand and Weissleder, 2010). One interesting fluorescence imaging application is the use of inherently fluorescent and NIR shifted mutated fluorescent proteins that have been transfected into cancer cells for the in vivo imaging of tumors (Hoffman, 2009). This technology has yielded important information about the spread of cancer by monitoring in vivo tumor cell trafficking. Numerous cellular targets such as small molecules, proteins, and various biological analytes have been targeted by NIR fluorophores and activatable probes for imaging cancer as well as other conditions (Hilderbrand and Weissleder, 2010). Although much success has been achieved in recent years, fluorescence imaging still suffers from poor signal due to tissue autofluorescence as well as a lack of anatomical resolution.

Nanoparticulate scaffolds have allowed for the multivalent attachment of dyes to increase their effective local concentration. Some of these materials also allow for multimodal imaging depending upon the particle composition or modification. Iron oxide nanoparticle-NIR fluorophore conjugates have been shown to be useful in tumor imaging with the aid of
A
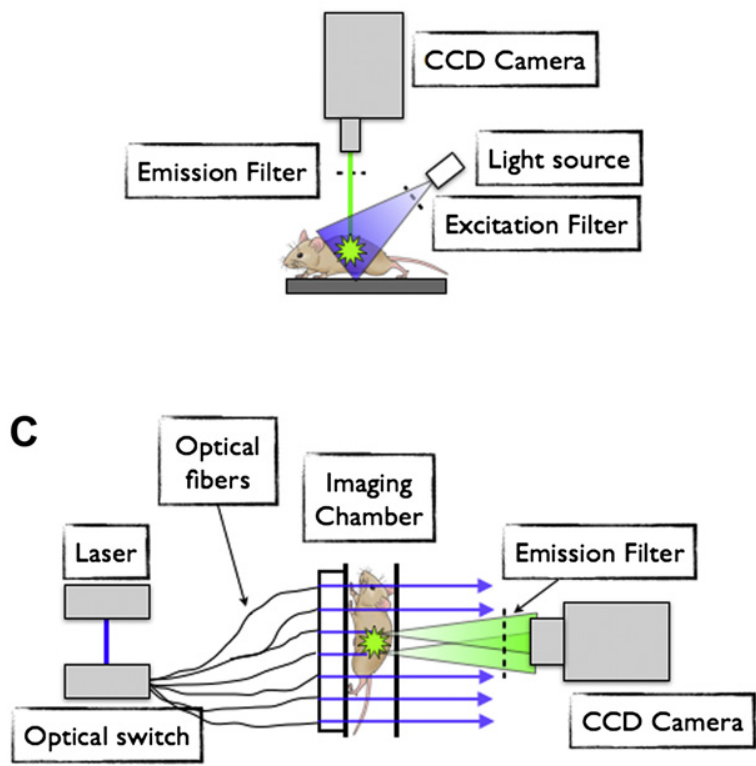

B
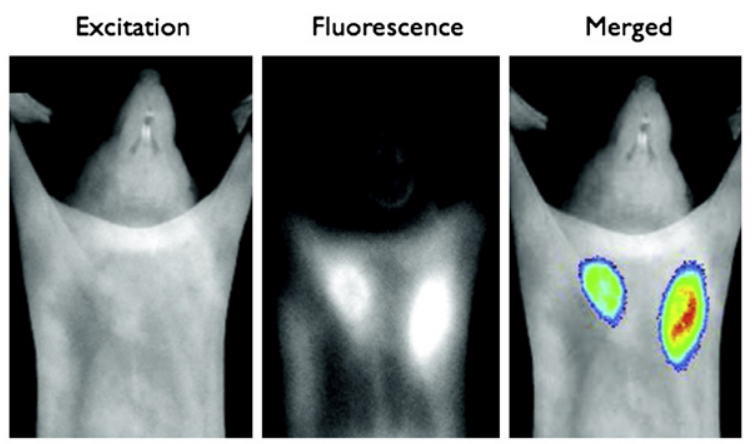

D
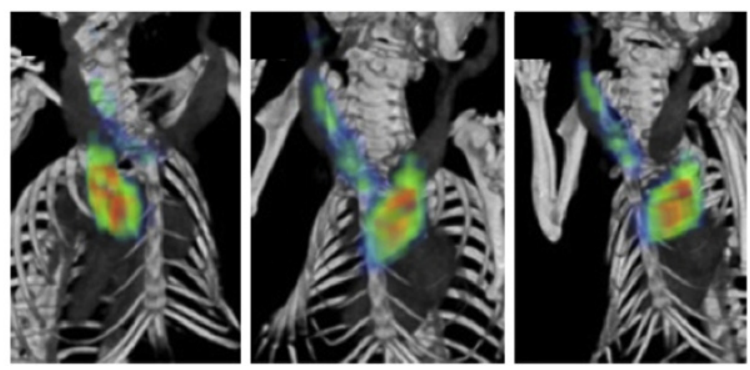

Figure 1 - Commonly utilized modalities for in vivo fluorescence imaging. A) Simplified schematic representation of a fluorescence reflectance imaging (FRI) system. B) FRI yields a planar image lacking information regarding the depth of the fluorophore. C) Simplified schematic representation of a fluorescence molecular tomography (FMT) system. D) FMT yields images that can be reconstructed in three dimensions, such as the hybrid FMT-CT image shown. Reproduced, in part, with permissions from Nahrendorf et al. (2009) and Ntziachristos, 2006. 
targeting ligands such as plasma clotting peptides that lead to enhanced tumor uptake (Simberg et al., 2007) and hepsin-targeted peptides for prostate cancer (Kelly et al., 2008). Quantum dots (QDs) are highly fluorescent particles that have found recent use in cancer imaging because of their versatility and brightness that can be easily tuned to different emission wavelengths (Biju et al., 2010). Hybrid magnetic-fluorescent QD nanoparticles have been shown to detect tumors in mice by both MRI as well as fluorescent imaging allowing for enhanced resolution as well as anatomical information (Park et al., 2008). Fluorescence imaging has become increasingly popular over the past decade particularly with advancements in FMT and is now a highly valuable resource in biological imaging.

\subsubsection{Ultrasound imaging}

Ultrasonic or ultrasound imaging involves the pulsation of high frequency sound waves by an acoustic transducer and the measurement of echoes or reflected sound as the waves enter material of different acoustical impedance (Kherlopian et al., 2008). A greater change in impedance leads to a more powerful echo and the time an echo takes to travel back to the probe can be used to calculate depth and help create a computer interpreted image of the scanned material (Jensen, 2007). Because of physical differences in tissues density, features such as blood flow, organs, and tumors may be distinguished by ultrasound imaging. Ultrasound is relatively inexpensive, as compared to other imaging techniques, but sensitivity is limited by the amount of tissue the sound waves must travel through. While ultrasound has shown use in imaging and tumor detection in the past (Mittelstaedt, 1980), comparisons with other techniques such as MRI demonstrate their superiority over ultrasound in terms of tumor imaging (Curati et al., 1988). Ultrasound was however found to be very useful for imaging vasculature, which has led to its use in imaging tumor angiogenesis as well as various cardiovascular conditions (Christiansen and Lindner, 2005). In order to increase the contrast in ultrasound images, ultrasound reflective agents, such as microbubbles or other nanoparticle-based contrast agents have been investigated (Kaufmann and Lindner, 2007; Rapoport et al., 2007) (Table 1).

\subsection{Enhanced permeability and retention (EPR) effect}

As imaging technologies continue to advance and become more sophisticated, there is more potential for the early detection of cancer. Standing in the way however, is the challenge of delivering a sufficient amount of agent to sites of cancerous tissue to allow for imaging to take place. Most current strategies involve the discovery and use of targeting moieties that bind specifically to unique features of different cancers. While this targeted approach will be discussed in later sections of this review, this section will deal with a passive tumor targeting phenomenon that was first described in the literature over 20 years ago that is known as the enhanced permeability and retention (EPR) effect.

The EPR effect describes the intrinsic ability of macromolecules and particles of a certain size to accumulate in solid tumor tissues (Iyer et al., 2006; Maeda, 2001; Maeda et al., 2000). The first study to demonstrate this concept showed that ${ }^{51} \mathrm{Cr}$ labeled proteins larger than $15 \mathrm{kDa}$ accumulated in tumors to a greater extent than small molecules and were retained for longer periods of time (Matsumura and Maeda, 1986). This effect was attributed to unique tumor vascular characteristics such as a rapidly increasing number of blood vessels, increased extravasation, as well as a poor lymphatic recovery system, resulting in significantly decreased re-uptake of macromolecules into the bloodstream (Matsumura and Maeda, 1986; Iyer et al., 2006). Thus, solid tumors could be selectively targeted exploiting inherent 'leaky' tumor vasculature. This effect has been compared to Paul Ehrlich's infamous 'magic bullet' concept and has been described as a gold standard for tumor targeting (Iyer et al., 2006; Maeda, 2001; Maeda et al., 2000).

The importance and potential impact of the EPR effect in tumor targeting has led to a significant interest in the factors

Table 1 - Recent reviews of nanoagents used in the imaging of disease.

\begin{tabular}{lll} 
Modality & \multicolumn{1}{c}{ Agent } & \multicolumn{1}{c}{ References } \\
\hline Magnetic resonance imaging & Nanoparticles containing gadolinium chelates & \multicolumn{1}{c}{$\begin{array}{l}\text { Aime et al. (2009) } \\
\text { Lin et al. (2009) } \\
\text { McCarthy and Weissleder (2008) } \\
\text { Lin et al. (2008) } \\
\text { Lin et al. (2009) }\end{array}$} \\
& Superparamagnetic iron oxide nanoparticles & Hamoudeh et al. (2008) \\
Nuclear imaging & Ting et al. (2009) \\
& Radionuclide-modified nanomaterials & Dancey et al. (2009) \\
Fluorescence imaging & & Xing and Rao (2008) \\
& Quantum dots & Santra et al. (2005) \\
& Jiang et al. (2010) \\
& Fluorophore-labeled nanoparticles & Santra et al. (2005) \\
Ultrasound imaging & Paneda et al. (2009) & Tran et al. (2007b) \\
& & Dayton et al. (2006) \\
& & Lanza et al. (2006)
\end{tabular}


that mediate this effect. Features that have been identified as EPR mediators include vascular permeability enhancing and vasodilating factors such as vascular endothelial growth factor, bradykinin, prostaglandins, and nitric oxide that are upregulated or present in larger quantities in tumors compared to normal tissue (Iyer et al., 2006). Anatomical differences in tumor vasculature also contribute, such as the lack of a smooth muscle layer surrounding the vessels that leads to increased blood flow volume to tumors during hypertension and hence more leakage of macromolecules into the surrounding tissue (Iyer et al., 2006). Other factors affecting tumor uptake by the EPR effect include molecular weight, which increases uptake with molecules above the renal threshold of $\sim 40 \mathrm{kDa}$, prolonged circulation time, and the diameter of the macromolecule or particle (Iyer et al., 2006; Maeda, 2001; Maeda et al., 2000). It has been demonstrated that particles with a diameter as large as $400 \mathrm{~nm}$ can extravasate into tumors using human colon cancer xenographs (Yuan et al., 1995). Further details, including the description of different attempts to augment the EPR effect by increasing certain factors can be read in a recent review (Iyer et al., 2006).

\subsection{Intrinsic targeting}

Utilizing general properties that differentiate cancerous tissue from normal tissue such as those outlined in the previous section to target tumors can be defined as intrinsic or passive targeting. Biocompatible nanoparticles comprise a significant amount of intrinsically targeted tumor imaging agents. By exploiting the inherent EPR effect properties, nanoparticles that are either composed of material that can be imaged itself, or that are conjugated in some way to imaging moieties have been used with great success in the identification of cancerous tissue using a variety of imaging modalities.

One example using MRI to image brain tumors has been demonstrated by Enochs et al. (1999). Human patients with recurrent brain tumors were administered two different types of contrast agents, dextran-coated iron oxide nanoparticles and a small molecule Gd chelate. While both were able to provide contrast enhanced MR images of the brain tumors, the nanoparticles were able to afford improved definition of the tumor margins over a longer period of time. The small molecule Gd chelate began to diffuse into the surrounding normal brain tissue after a period of hours, thus blurring the tumor margins, but the iron oxide nanoparticles remained localized to tumor tissue for several days. This study not only demonstrated the ability of iron oxide nanoparticles to image human brain tumors, but also yielded an advantage of using passively targeted nanoparticles as opposed to small molecule imaging agents.

Another clinical example demonstrating the use of superparamagnetic iron oxide nanoparticles in MRI-based cancer imaging was in the detection of prostate cancer metastases (Harisinghani et al., 2003). Human patients with prostate cancer were imaged before and $24 \mathrm{~h}$ after injection with the nanoparticles to compare the T2-weighted images. The nanoparticles were taken up into healthy lymph nodes, but not by lymph nodes containing metastases. This results in a difference in MR signal. The difference in T2-weighted signal can be seen in Figure 2 in which two nodes before injection show similar contrast, but after injection, the metastatic node shows a high signal compared to the decreased signal in the normal node. This method was found to be very accurate in diagnosing lymph node metastases that were otherwise undetectable using other techniques.

Passive tumor targeting has also been demonstrated using fluorescent imaging with QDs. QDs composed of a core of CdSe/ZnS with an amphiphilic polymer coating were found to accumulate in human prostate cancer cells implanted into mice (Gao et al., 2004). Tumor accumulation was sensitive to functional groups present on the polymer coating. While carboxylic acids yielded no tumor accumulation, polyethylene glycol (PEG) functionalized QDs were retained by the tumor. This was explained by the PEG groups preventing opsonization of blood-borne proteins, such as albumin, thereby promoting a longer blood circulation time and allowing for more tumor accumulation of the PEG coated QDs as opposed to the carboxylic acid QDs which were said to be excreted more rapidly by the reticuloendothelial system.

Newer studies have combined imaging technologies such as the development of hybrid magnetic-QD nanoparticles for simultaneous MR and fluorescent tumor imaging. In 2008, Park et al. reported the synthesis and in vivo analysis of such particles encapsulated in PEG coated micelles (Park et al., 2008). These magnetofluorescent micelles were injected into tumor bearing mice and imaged $20 \mathrm{~h}$ post-injection. The hybrid nanoparticles were shown to accumulate in the tumors as seen in vivo by pre and post-injection fluorescent images of the mice. Excised tumor tissue after the $20 \mathrm{~h}$ incubation period was imaged with both an MRI scanner and NIR optical imaging system to afford the comparative images of PBS and nanoparticle treated mice. While the MRI image alone shows significant increase in signal of the tumors from nanoparticle injected mice, the fluorescent image corroborates this result and is even more pronounced with little to no signal observed in tumors from PBS injected mice.

Nuclear imaging of passively delivered nanoparticulate radionuclides has also been conducted using an ${ }^{111}$ In complex encapsulated in PEG coated liposomes (Harrington et al., 2001). Patients with different types of cancer were injected with the liposomes and were imaged using wholebody gamma cameras and an SPECT scanner at intervals up to 10 days post-injection. Scans revealed that the liposomes were able to accumulate in several different types of solid tumors effectively delivering the radionuclide to afford images of the cancerous tissue. Liposomal uptake by the different types of tumors revealed that head and neck cancers accumulated more of the radionuclide $(33.0 \pm 15.8 \%$ injected dose (ID) $/ \mathrm{kg}$ of tumor) than lung (18.3 $\pm 5.7 \% \mathrm{ID} / \mathrm{kg})$ and breast $(5.3 \pm 2.6 \% \mathrm{ID} / \mathrm{kg})$, but no conclusive data was determined as to why this was so.

These selected examples only represent a few of the many studies that have been conducted using the passive targeting of solid tumors with nanoparticulate systems, but they demonstrate the utility that exploiting the EPR effect has in cancer imaging. This 'non-targeted' approach has given researchers a way to localize agents to tumors without knowing more detailed anatomical or physiological information about the specific type of cancer. Despite the success of this strategy, more specific targeting is often required. The majority of 

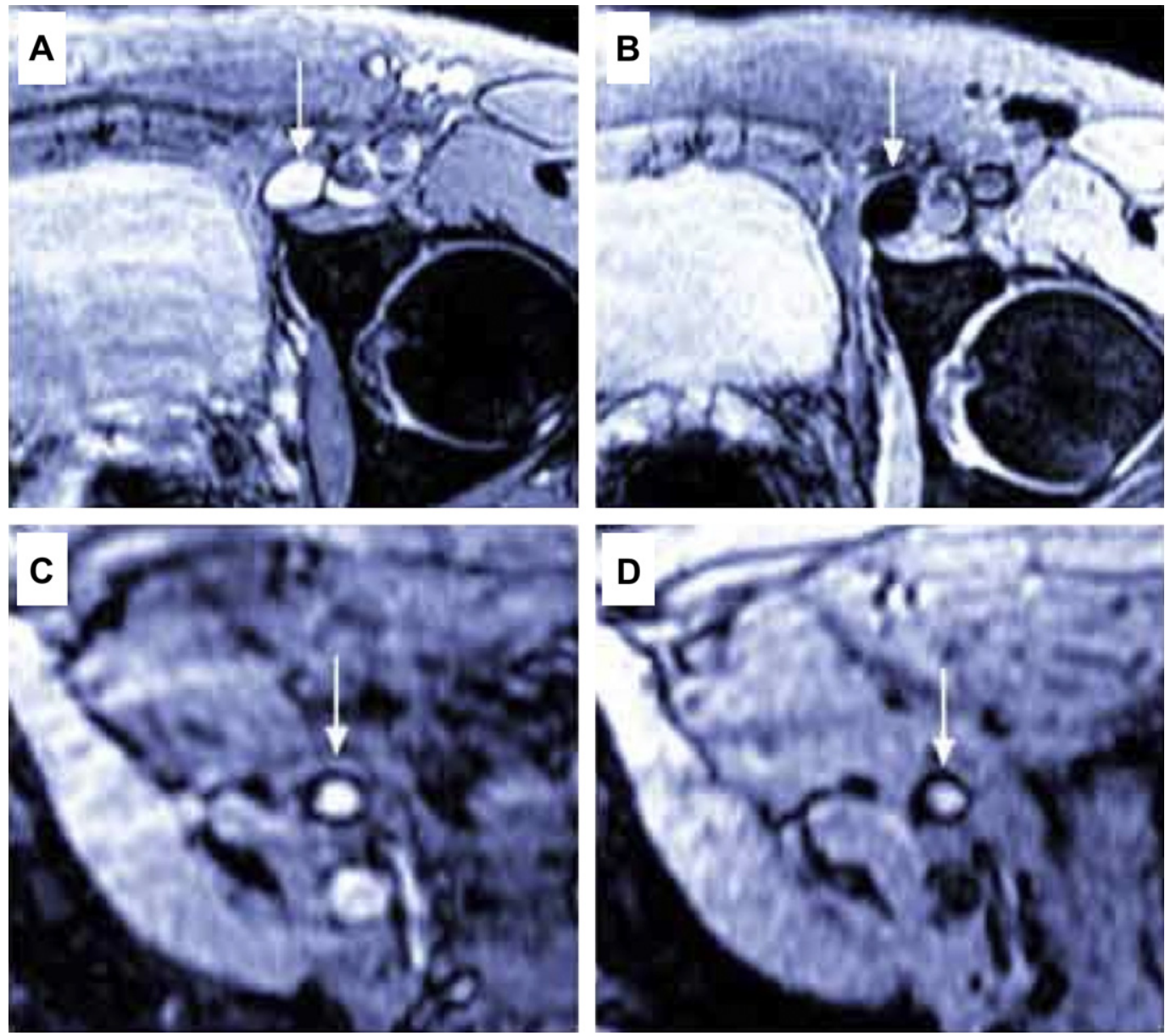

Figure 2 - MRI nodal abnormalities in two patients with prostate cancer. As compared with conventional MRI (A), MRI obtained $24 \mathrm{~h}$ after the administration of superparamagnetic nanoparticles (B) shows a homogeneous decrease in signal intensity due to the accumulation of the nanoparticles in a normal lymph node (arrow). Conventional MRI shows a high signal intensity in an unenlarged iliac lymph node completely replaced by tumor (arrow in C). Nodal signal intensity remains high (arrow in D). Reproduced, in part, with permission from Harisinghani et al. (2003).

current nanoparticulate cancer imaging research uses many other biological features found in specific types of cancer to more selectively target and image tumor tissue, which will be discussed in the following sections.

\section{Identification of affinity ligands for targeted imaging}

Nanoparticulate imaging agents have been targeted to sites of interest via a number of different affinity ligands. This section will highlight the most commonly utilized ligands, and discuss their potential advantages and disadvantages.

\subsection{Antibodies}

Antibodies are important components of the immune system, yet they have also found applicability in the treatment of a number of diseases, including cancers. In particular, monoclonal antibodies (mAbs) belong to a class of molecules that are specifically engineered to bind to targets of interest with high affinity. The use of mAbs to target a variety of receptors present in tumors has become widespread, as mAbs can function in a number of ways in order to bring about a therapeutic effect, including by directly attaching to the tumor cells to increase their detectability by the native immune system, by inhibiting growth factors, and by allowing for the targeted delivery of therapeutic radionuclides for radioimmunotherapy. Due to the extraordinary specificity and affinity demonstrated by mAbs for their targets of interest, they have also been utilized for the detection of diseased tissues via the conjugation of a number of different imaging agents. The two most prevalent examples are mAbs routinely utilized in the treatment of cancers with upregulated epidermal growth factor receptors.

The epidermal growth factor receptor (EGFR, ErbB-1) is a transmembrane glycoprotein, and a member of a closely related tyrosine kinase receptor family. It is present in normal cells, but mutations may lead to its upregulation in many cancers, which can be correlated with a poor prognosis and low survival rate (Herbst, 2004). Overexpression of EGFR results in unchecked cell division in the presence of epidermal 
growth factor. Thus, a significant amount of research has focused upon the generation of agents capable of interfering with EGFR function by blocking the receptor-binding site, including mAbs, such as cetuximab and panitumumab (Saltz et al., 2006).

In order to develop a methodology for the fluorescencebased characterization of EGFR status in a breast cancer, Wang et al. (2009) have labeled cetuximab with the NIR fluorescent dye Cy5.5. The EGFR specificity of the antibody-based probe was initially examined by flow cytometry and laser confocal microscopy in MDA-MB-231 cells, which overexpress EGFR, and MCF-7, which do not. As expected, the probe demonstrated appreciable binding to the receptor expressing cells ( 9-fold higher than non-EGFR expressing cells), which could be abrogated by preincubation of the cells with an anti-EGFR antibody. In vivo experiments were performed in immunodeficient mice bearing MDA-MB-231 or MCF-7 tumors. After injection of the cetuximab-Cy5.5 probe, wholebody and region-of-interest fluorescence images were collected and analyzed. As was demonstrated in vitro, agent uptake in EGFR-positive tumors was readily observed (Figure 3). Immunohistochemical analysis of the tumor tissues was further utilized to correlate the EGFR expression in MDA-MB-231 and MCF-7 tumors.

\section{MDA-MB-231}
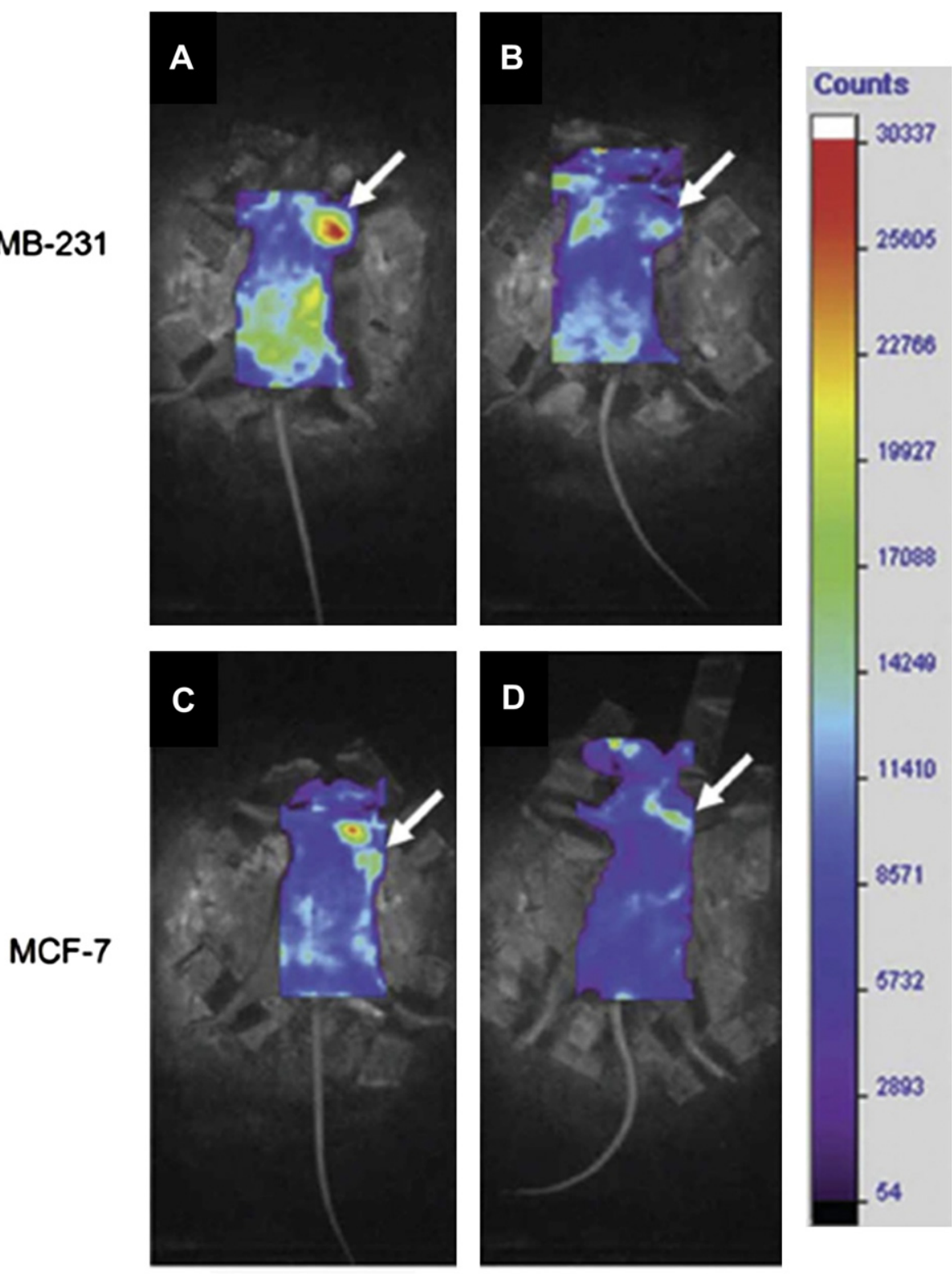

Control
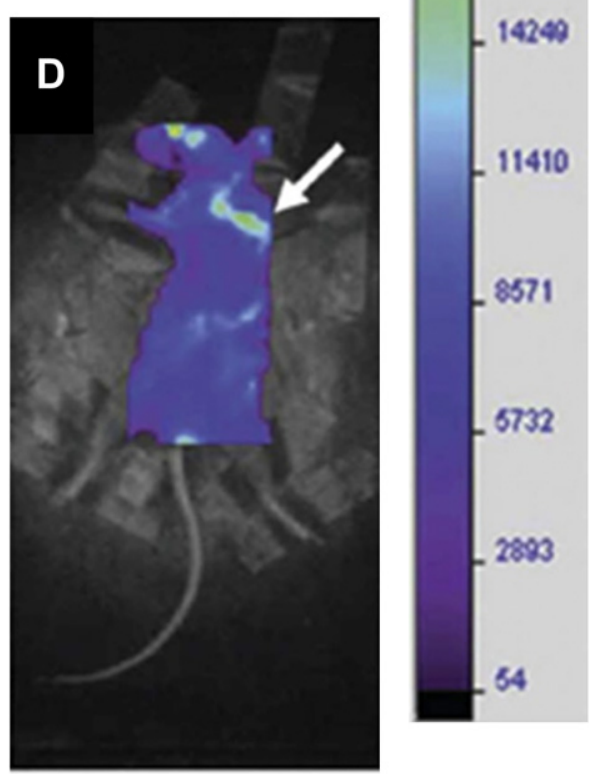

\section{Block}

Figure 3 - In vivo near-infrared imaging of EGFR-positive tumors. MDA-MB-231 or MCF-7 cells were injected into the left chest mammary gland fat pad of female athymic nude mice. Representative whole-body NIR images of MDA-MB-231 and MCF-7 xenograft mice at $24 \mathrm{~h}$ postinjection of cetuximab-Cy5.5 conjugate are shown. A and C) Fluorescence signal is clearly visualized in the left thoracic tumor region of MDA-MB-231 and MCF-7 xenografts. B and D) Blocking experiment indicates an apparent decrease of fluorescent signals by pre-injection of excess cetuximab (tumors indicated by arrows). Reproduced with permission from Wang et al. (2009). 
EGFR targeted nanoparticles have been developed for in vivo targeting of different types of tumors via immobilization of anti-EGFR antibody (ScFvEGFR) on the nanoparticles. Yang et al. (2009a) have developed ScFvEGFR surface functionalized iron oxide nanoparticles for in vivo MR imaging of pancreatic tumor cells. The targeted nanoparticles were synthesized with an amphiphilic triblock polymer coating to allow for surface functionalization. ScFvEGFR was immobilized on the amine bearing nanoparticles via carbodiimide mediated coupling methods to give the targeted nanoparticles with a $10 \mathrm{~nm}$ core size. For in vivo studies, PaCa-2 pancreatic tumor bearing nude mice were intravenously injected with non-targeted and targeted nanoparticles and MRI scans of the mice were recorded at intervals from $5 \mathrm{~h}$ to $30 \mathrm{~h}$ after the injection. A 4.8 fold MRI signal enhancement was seen between the tumor of the mouse that received targeted nanoparticles and the mouse that was administered the non-targeted nanoparticles. For further verification, mice were sacrificed for histological studies, which confirmed the selective uptake of targeted nanoparticles by tumor cells.

The HER-2/neu (HER-2) oncogene is another closely related member of the epidermal growth factor receptor family (Hung and Lau, 1999). This receptor is known to be upregulated in different types of human cancers including breast, ovarian, lung, gastric, and oral cancers. Enhanced malignancy and poor survival rates of breast cancer patients have been associated with the upregulation of HER-2. In addition, the upregulation of HER-2 seems to impart upon the tumors a degree of chemoresistance. Suppression of the malignant phenotypes of HER2/neu-overexpressing cancer cells through repression of HER-2/neu was observed in many studies indicating HER-2 can serve as an excellent target for developing anticancer agents specific for HER-2 upregulated cancer cells.

Sampath et al. (2007) have reported the dual-labeling of a HER-2 mAb, trastuzumab, with an ${ }^{111}$ In chelate and a fluorescent dye, IR800, in order to enable multimodal imaging of Her-2 overexpression. In vitro, the conjugate was readily able to differentiate between SKBr3 (HER2-positive) and MDA-MB-231 (HER2-negative) breast cancer cells. The conjugate was next assayed in vivo in athymic mice bearing SKBr3 tumors. Forty eight hours after agent injection, the mice demonstrated significant uptake in the tumors by both SPECT and fluorescence imaging, which could be reduced by pre-injection of trastuzumab.

While antibodies offer exceedingly high target selectivity and binding affinities, they also possess several drawbacks. This includes potential immunogenicity, which may be overcome by the engineering of the antibodies to possess humanized or chimeric properties, thus evading immune detection. Antibody production and isolation is also difficult and laborious, which leads many researchers to explore the use of alternative affinity ligands, such as those detailed below.

\subsection{Peptides}

As compared to antibodies, peptide-based targeting ligands offer several advantages, such as a lack of immunogenicity. They can also be readily synthesized at a fraction of the cost of antibody production. However, peptides incur a number of drawbacks, including lower target affinities, increased chance of non-specific binding, and an increased likelihood of proteolytic cleavage. Some of these issues may be improved upon by displaying the peptides multivalently, thereby increasing the effective binding affinity, or by including $\mathrm{D}$-amino acids, which evade proteolysis.

Peptide-based targeting ligands are identified via a number of different methods. Most commonly, they are gleaned from the binding regions of proteins for the target of interest. In these cases, peptide libraries are often synthesized in order to delineate the optimal peptide sequence, and may utilize single amino acid mutations in order to fully understand the binding of the resulting peptide to its partner.

Another methodology that is commonly used is phage display. Phage display is a technique utilized in drug discovery for efficient and rapid screening of peptides possessing specific binding to targets of interest (McCarthy et al., 2007). In this methodology, bacteriophage, viruses that only infect bacteria, are genetically modified to express multiple copies of a single peptide on their surface, yet form a library containing over $10^{9}$ different sequences. This library can be utilized in a number of different ways. The simplest is based upon screening against purified targets immobilized on plates. After exposure to the phage, the plate is washed to remove any unbound phage, while the bound phage are isolated, expanded, and re-plated with the target (Figure 4). This is repeated a number of times until a consensus sequence is reached. One of the main drawbacks of this methodology is that the target molecules are not in their native state and may not truly represent the in vivo environment. Several improvements to phage display screening have been made, including cell-based screening of peptides and screening under flow conditions which may give phage with increased binding affinities. In vivo phage display has recently been developed to obtain disease-specific clones. In this procedure, phage clones can be selected based upon their potential to reach target cells.

Peptide ligands can also be identified by utilizing in silico data that is available in electronic databases (McCarthy and Weissleder, 2008). Even though there are many existing electronic resources and databases for the identification of protein sequences, there are only few similar resources to find efficient peptide targeting ligands. For quick identification of potential target peptides, PepBank, a searchable database has been created, based on sequence text mining and public peptide data sources (http://pepbank.mgh.harvard.edu). This database is currently comprised of over 21,000 entries.

While there are countless peptide sequences being utilized for the targeting of diagnostic and therapeutic moieties, one in particular, the tripeptide arginine-glycine-aspartate (RGD), has been used widely by a multitude of researchers. RGD binds to $\alpha_{v} \beta_{3}$ integrin, which is upregulated in activated endothelial cells, especially during inflammation or angiogenesis, both of which are present in cancers (Cairns et al., 2003; FeldingHabermann, 2003). While initial research focused upon the utility of the peptide itself in the treatment of cancers, subsequent work has utilized it for the delivery of both therapeutic and imaging agents to tumors.

One example, by Cheng et al. (2005), describes the conjugation of Cy5.5 to mono-, di-, and tetra-meric RGD peptides and the testing of each conjugates targeting ability to a U87MG glioblastoma xenograft model. Overall, the goal of the study was to examine the effect multivalency has on avidity and 


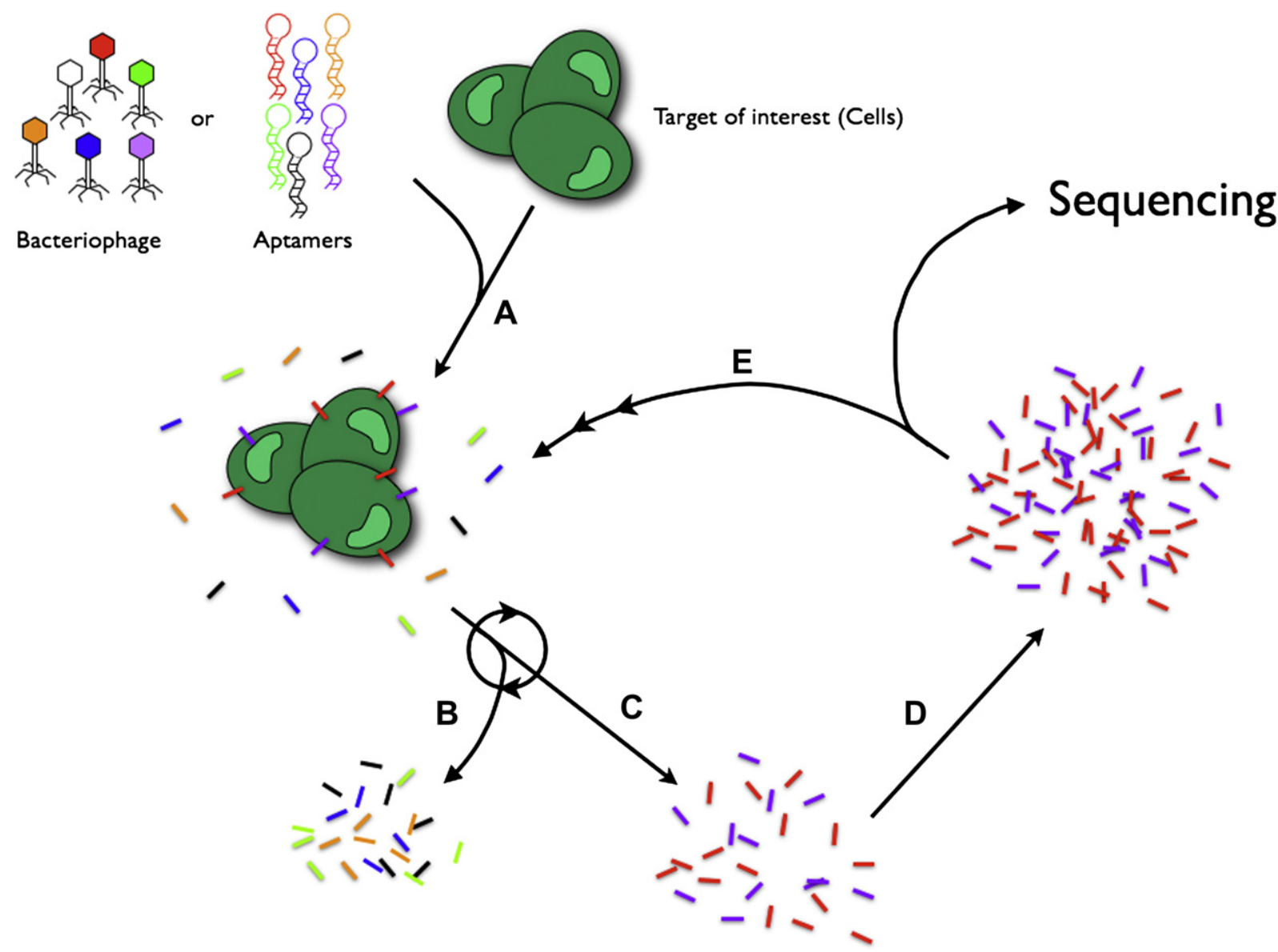

Figure 4 - Generalized screening repertoire for the discovery of targeting peptides and aptamers. A) Libraries of aptamers or bacteriophage are plated with the target of interest. This may be purified protein immobilized on a plate or cell-based. B) Non-bound sequences are removed from the target by repetitive washing. C) Bound sequences are isolated from the target of interest. D) The isolated aptamers and phage are amplified by polymerase chain reaction (PCR) or via infection of E. coli, respectively. E) The expanded aptamers or phage are either subjected to additional rounds of screening, or are sequenced in order to identify the targeting ligand.

efficacy of the peptide towards tumor binding. As was hypothesized, the authors found that while all dye-RGD conjugates showed specific uptake by glioblastoma both in vitro and in vivo, the tetrameric conjugate demonstrated the highest avidity for $\alpha_{v} \beta_{3}$ integrin.

Lee et al. (2008) have developed a multifunctional RGD bearing iron oxide (IO) nanoparticle probe for PET/MRI imaging of human glioblastoma cancer cells. Iron oxide nanoparticles were initially coated with polyaspartic acid, to which was conjugated 1,4,7,10-tetraazacyclododecane-1,4,7,10-tetraacetic acid (DOTA) to serve as a chelating agent for the ${ }^{64} \mathrm{Cu}$ radionucleotide. DOTA-labeled particles were then decorated with RGD via well-established activation-conjugation methods. In the final step of the synthesis, DOTA-IO-RGD nanoparticles were treated with ${ }^{64} \mathrm{Cu}$ under slightly acidic conditions to obtain ${ }^{64} \mathrm{Cu}$-DOTA-RGD-IO nanoparticles. The targeted nanoparticles were injected into mice bearing U87MG tumors, and were followed by PET/MRI imaging. The imaging studies revealed that the U87MG tumor could be clearly visualized with ${ }^{64} \mathrm{Cu}$-DOTA-IO-RGD nanoparticles, whereas the non-targeted ${ }^{64} \mathrm{Cu}$-DOTA-IO particles exhibited very low non-specific tumor uptake.

\subsection{Aptamers}

Aptamers are short oligonucleotides (15-40 bases) that are identified via selection processes similar to phage display, termed systematic evolution of ligands by exponential enrichment (SELEX) (Ellington and Szostak, 1990; Tuerk and Gold, 1990), that have potential uses as both therapeutic and targeting moieties (Farokhzad et al., 2006). Aptamers are highly specific for their targets and possess binding affinities rivaling antibodies, largely due to the ability of the molecules fold into complex three-dimensional structures (Levy-Nissenbaum et al., 2008). Aptamers have several advantages over antibodies, including the fact that they can be synthesized via solid-phase methodologies, and display minimal immunogenicity. Unmodified aptamers possess several deleterious properties, including rapid blood clearance, largely due to nuclease degradation, yet methodologies have been developed to include subunits such as $2^{\prime}$-fluorine-substituted pyrimidines and PEG linkages in order to circumvent these difficulties.

As mentioned above, aptamers are identified through the SELEX procedure (Figure 4), in which a combinatorial nucleic acid library is initially created consisting of random 
nucleotide sequences flanked by fixed sequences as primer binding sites (Guo et al., 2008). The nucleic acid library is initially incubated with targets of interest, and the unbound aptamers are removed from the bound ones through washing. The bound aptamers are then eluted from the binding partners, and are amplified through polymerase chain reaction (PCR). The whole procedure is repeated several times until highly enriched sequences are obtained. The nucleic acids obtained are subjected to DNA sequencing and examined for binding affinity. Modified SELEX strategies using whole living cells as targets has been termed as cell-SELEX, and can be applied for designing more effective aptamer-guided materials thereby enhancing intracellular delivery (Guo et al., 2008). The object of this procedure is to develop aptamers that will bind to or be internalized by a specific cell population.

\subsection{Small molecules}

The detection of diseases using small molecule-modified materials has become increasingly popular as an alternative targeting strategy. This is due to the seemingly infinite diversity that small molecules possess in their structures and properties, which is partly facilitated by the use of diversity-oriented synthesis (DOS), allowing for rapid access to libraries of diverse molecules with different functionalities starting from common framework (McCarthy and Weissleder, 2008). In cases where small molecules themselves may not demonstrate efficacious binding to targets, their small size allows for incorporation of a large number of ligands per nanoscaffold, thereby increasing the effective target affinity via multivalency.

We have previously developed a library of 146 diversely functionalized small molecules (e.g. amines, alcohols, carboxylic acids, sulfhydryls or anhydrides) which were used to label magnetofluorescent iron oxide nanoparticles (Weissleder et al., 2005). The resulting library was screened against five different cell lines including human umbilical vein endothelial cells (HUVEC), resting primary human macrophages, granulocyte macrophage colony stimulating factor-stimulated primary macrophages, a human macrophage-like cell line (U937), and human pancreatic ductal adenocarcinoma cells (PDAC, PaCa2). The cell uptake was measured by fluorescence microscopy and flow cytometry. As expected, cellular uptake of the agents varied greatly, but allowed for the discovery of a number of hits for materials that were preferentially taken up by activated versus resting macrophages, and those which were preferentially internalized by the PDAC cells over all other cells. When the latter materials were tested in vivo, the PaCa-2 tumors demonstrated significant localization of the agents by both fluorescence reflectance imaging and histology.

In order to get a better understanding of the role of multivalency in the targeting of nanomaterials with small molecules Tassa et al. (2010) have used surface plasmon resonance (SPR) to characterize the binding of these agents to targets of interest (Figure 5). Using a library of structurally related small molecules for the same protein, FKBP12, with dissociation constants $\left(K_{D}\right)$ varied over a 4500 -fold range, the authors conjugated the small molecules to magnetofluorescent nanoparticles. Initial investigations focused on the loading density of the FKBP12-GST (glutathione S-transferese) fusion protein on the SPR sensor chip, as many nanoparticle preparations did not show appreciable dissociation from the chip loaded with a higher density of protein, negating the calculation of binding constants. The authors thus reduced the loading of protein to lower densities (1 protein per $648 \mathrm{~nm}^{2}$ ), a number that closely resembles physiologically relevant receptor densities. For both small molecules and their nanoparticle conjugates, association rate $k_{\mathrm{a}}$, dissociation rate $k_{\mathrm{d}}$, and dissociation constant $K_{\mathrm{D}}$ $\left(k_{\mathrm{d}} / k_{\mathrm{a}}\right)$ for the interaction of FKBP12 were determined. All conjugates revealed significant decrease in $k_{\mathrm{d}}$, whereas changes in $k_{\mathrm{a}}$ varied greatly, as compared to the free molecules. Overall, the multivalent attachment of small molecules to the nanoscaffold surface resulted in the similar $K_{D}$ for all conjugates, even though they display marked differences in kinetics (Figure 5C). The authors hypothesize that the generality of this approach can be utilized to quickly screen nanoparticle libraries as it does not require the development of cell-based assays for each target.

While the above research focused upon the identification of novel targeting ligands, a number of small molecules have been used routinely for the delivery of agents, including folate and carbohydrates. Folic acid is a water-soluble vitamin (vita$\min \mathrm{B}_{6}$ ) whose tetrahydrofolate form is of biological importance. Folate is responsible for the synthesis of DNA and for the repair of RNA. Folate is also essential in humans for rapid cell division and growth especially during the development of embryos. In cancers, where cell division is unchecked, the folate receptor may be upregulated in order to provide cells with the required amount of folate. Thus, folic acid has been used as a delivery vehicle for a number of imaging and therapeutic agents to tumors (Low and Kularatne, 2009). Clinically relevant examples include ${ }^{111}$ In-DTPA-folate, EC20 (a folate conjugate of ${ }^{99 \mathrm{~m}} \mathrm{Tc}$ ), EC17 (a folate-linked fluorescent hepten) and EC145 (a folate conjugate of diacetylvinylblastine hydrazide) that have been examined for clinical imaging and therapy purposes.

Carbohydrates are another broad class of small molecules that have shown applicability as targeting ligands due to their biocompatibility and their ability to be selectively recognized by cell surface receptors, such as lectins (Zhang et al., 2010). Whereas glycoproteins are endogenously expressed in a number of tissues, certain cells have been shown to upregulate carbohydrate receptors in response to inflammation, such as selectins on endothelial cells. One location that expresses a significant number of carbohydrate receptors is the liver. In particular, hepatocytes express the asialoglycoprotein receptor (ASGP-R) which readily binds galactose, and thus may serve as a means to ameliorate liver specific drug delivery (Zhang et al., 2010).

\section{Targeted nanomaterials}

Given the variety of different cancers and the multitude of agents designed for their detection and treatment, it would be impossible to enumerate the vast majority of them in this review. Thus, we will focus upon the synthesis and utility of nanoagents targeted to the cancers with the highest incidence amongst men and women, prostate and breast cancers, respectively. 
A
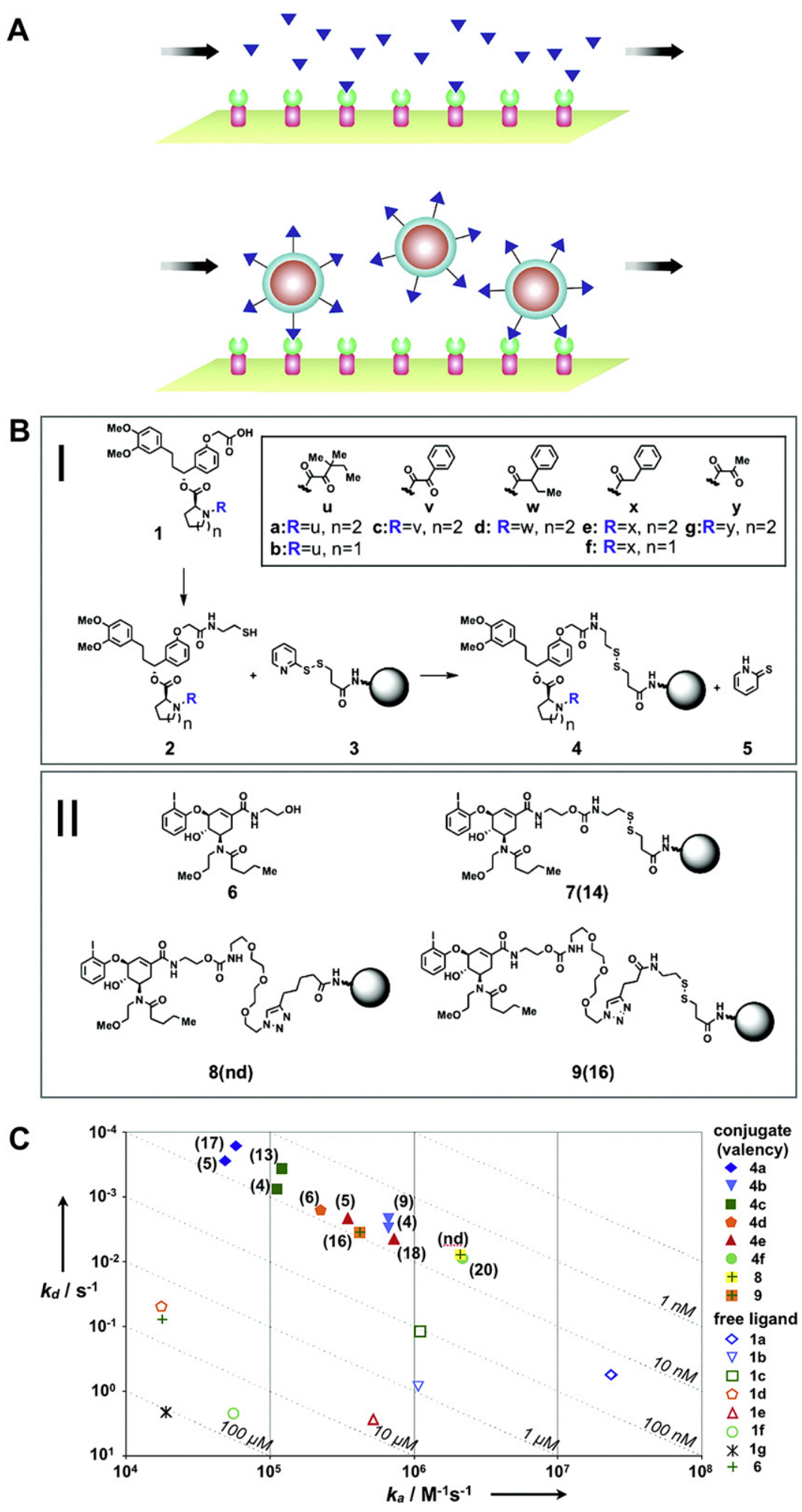

Figure 5 - Surface plasmon resonance (SPR) characterization of the binding of small molecule-modified nanoagents to their target protein. A) Schematic representation of SPR for free small molecules (top) and those bound multivalently to nanoparticles (bottom). B-I) Conjugation of a series of synthetic derivatives of FK506. B-II) Conjugation of a small molecule that binds aurora A kinase. C) Rate maps summarizing binding affinity and kinetics. Different combinations of $k_{\mathrm{a}}$ and $k_{\mathrm{d}}$ that result in the same $K_{\mathrm{D}}$ are indicated by dashed lines. Data for free ligands are depicted by open symbols; for each ligand, the corresponding nanoparticles are depicted by a solid symbol of the same shape, with conjugation valency listed in parentheses next to the solid symbol. Reproduced with permission from Tassa et al. (2010). 


\subsection{Targeted nanomaterials for detection of breast cancer}

Breast cancer is the most common type of cancer among women (American Cancer Society, 2009). It was estimated that in $2009,15 \%$ of cancer related deaths in women were due to breast cancer. This mortality rate is due, in part, to the high rate of tumor metastasis to bone and lymph nodes that develops during the progression of the disease in patients. Thus, early diagnosis is crucial to improve the survival rates of breast cancer patients.

To this end, much research has focused on the development of biomarker-targeted probes. Recently, the urokinasetype plasminogen activator (UPA) receptor, which is over expressed in 60-90\% of invasive breast cancer tissues (Han et al., 2005), was targeted by Yang et al. (2009b). uPA is an important target, as increased levels are a sign of tumor metastasis since the interaction of uPA with its cellular receptor advances angiogenesis and metastasis of the tumor (Fisher et al., 2000). Initially, uniform sized (10 $\mathrm{nm}$ core size) iron oxide nanoparticles were coated with an amphiphilic polymer bearing carboxylic acid side groups, some of which were subsequently modified with short polyethylene glycol chains to reduce non-specific binding and uptake by healthy tissues. The rest of the carboxyl groups were activated with a carbodiimide for conjugation of the amino terminal fragment of uPA. For imaging purposes, Cy5.5 was conjugated to a fragment peptide via the free thiol groups of the peptide before conjugation to iron oxide nanoparticles. The resulting nanoagent was tested in mouse mammary tumor 4T1 cells, expressing high levels of the UPA receptor on their surface. As a negative control, a human breast cancer cell line that lacks the uPA receptor was employed (T47D). Incubation of the cells with the agent for $3 \mathrm{~h}$ resulted in internalization by the $4 \mathrm{~T} 1$ cells but not by T47D cells. Later, mice bearing 4T1 tumors were used for in vivo NIR optical imaging studies (Figure 6). After injection of the agent, the 4T1 tumors were imaged at several time points and demonstrated increased signal intensity for up to $48 \mathrm{~h}$. The tumor selectivity of the uPA receptor-targeted particle was also demonstrated by MR imaging of the nanoparticle localization in the animal model.

As was discussed above, herceptin (trastuzumab) is a monoclonal antibody targeted to the HER-2/neu receptor, which is over expressed in ovarian and breast cancer cells (Tran et al., 2007a). Thus, numerous trastuzumab-modified nanoagents have been reported. For example, Chen et al. have used trastuzumab-modified superparamagnetic iron oxide (SPIO) nanoparticles for the in vivo and in vitro imaging of breast cancer (Chen et al., 2009). Synthesis of the HER2/neu targeted-SPIO nanoparticles started with coating of the nanoparticles with dextran followed by crosslinking with epichlorohydrin to generate cross-linked iron oxide (CLIO) nanoparticles. Treatment of the CLIO with 2,2'-(ethylenedioxy)bisethylamine (EDBE) resulted in amine functionalized nanoparticles (CLIO-EDBE). In the final step of the synthesis, trastuzumab was conjugated via its free carboxylic acid to the surface of the CLIO-EBDE via stable peptide bond to give the desired trastuzumab-modified nanoparticles with core size $3.5 \pm 0.3 \mathrm{~nm}$ and high stability in the wide range of $\mathrm{pH}$ from 4 to 10 .
The HER-2 targeted nanoparticles were next examined in vitro and in vivo. HER-2/neu receptor positive (BT-474, SKBr3, MDA-MB-231, MCF-7) and negative (KB) cell lines were incubated with $0.5 \mu \mathrm{mol} /$ well of the trastuzumab-modified nanoparticles followed by treatment with IgG-fluorescein isothiocyanate. The in vitro studies clearly demonstrated that the nanoagent not only targets breast cancer cells but can also differentiate the expression levels of HER-2/neu in the cell lines. For the in vivo studies, mice were initially inoculated with SKBR-3 or KB cells in order to give the appropriate tumors. Trastuzumab-targeted nanoparticles were intravenously injected $(20 \mu \mathrm{mol} / \mathrm{kg})$ and the mice were imaged by MRI. As was demonstrated in vitro, the SKBR-3 tumor demonstrated significant agent binding as compared to the KB tumor, as exemplified by the darkening of the tumor in the $\mathrm{T}_{2}$-weighted image.

Another example of an EGFR family-targeted nanomaterial for the imaging of breast cancer is an anti-EGFR antibody bearing quantum dot (QD). It is known that EGFR can be over expressed in breast cancers, as we described above, and can thus serve as targeted biomarker for imaging. Yang et al. (2009a) have synthesized EGFR targeted-QDs by employing a novel conjugation strategy. The free amine group of a modified nickel (II) nitrilotriacetic acid (Ni-NTA) was covalently conjugated to carboxyl end of the QDs via a stable amide bond. Treatment of Ni-NTA-QDs with histidine tagged ScFvEGFR gave stable Ni-NTA-QDs-ScFvEGFR conjugates via interaction of Ni-NTA with histidine. Incubation of breast cancer cell lines known to overexpress EGFR (4T1 and MDAMB-231) with the Ni-NTA-QDs-ScFvEGFR clearly showed that the targeted agent selectively bound to EGFR expressing cell surface, while non-targeted QDs demonstrated negligible binding to breast cancer cells.

Peptide-based affinity ligands have also been utilized for the detection of breast cancer. Recently, Li et al. (2009) have published octreotide (OCT)-conjugated iron oxide nanoparticles targeting somatostatin receptors (SSTRs). SST is a regulatory peptide for which most endocrine tumors express receptors in much higher densities than on healthy cells. OCT, an analog of SST, has the ability to bind to a number of subtypes of SSTRs, including $\mathrm{SSTR}_{2}, \mathrm{SSTR}_{3}$ and $\mathrm{SSTR}_{5}$. SSTR-targeting nanoagents were prepared by initially coating the nanoparticle core with a dicarboxylic acid terminated PEG, followed by the covalent conjugation of the peptide to the carboxylates. The targeted nanoagent was initially investigated in MCF-7 breast carcinoma cells. In vitro MR imaging results demonstrated that the cells cultured with agent displayed substantially lower $T_{2}$ values than the cells cultured with non-targeted particles, a result that is associated with the increased iron oxide content of the cell. MCF-7 breast cancer tumor bearing nude mice were then utilized for in vivo examination of particle localization. Mice were injected with either the targeted or untargeted agents and imaged at $6 \mathrm{~h}$ post-injection. The targeted agent demonstrated a significant decrease signal in $\mathrm{T}_{2}$-weighted images, as compared to the pre-contrast image. The untargeted nanoparticles also demonstrated a slight decrease in signal, likely due to non-specific tumor delivery via the EPR effect, yet not to the same degree as the targeted particles. 
A
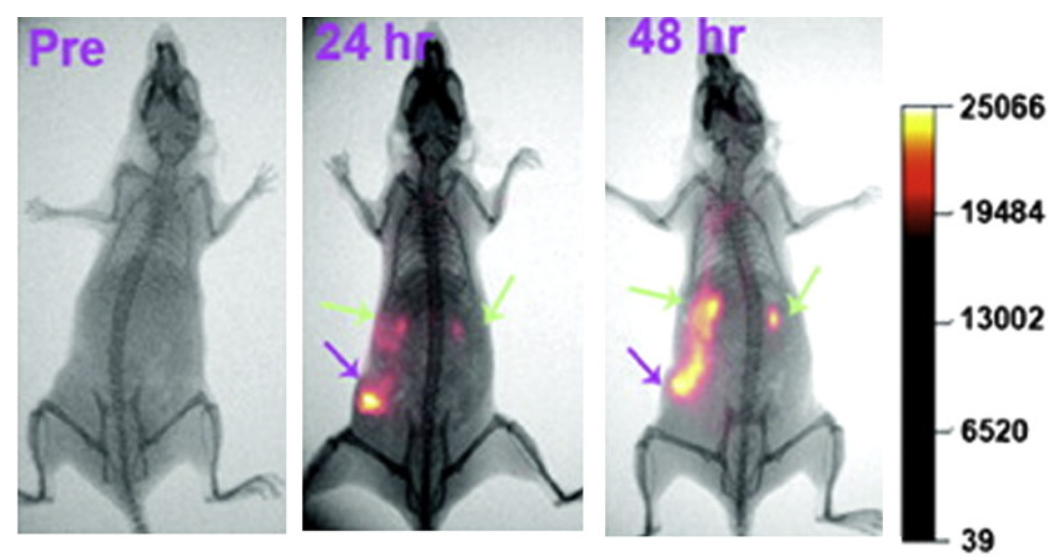

B
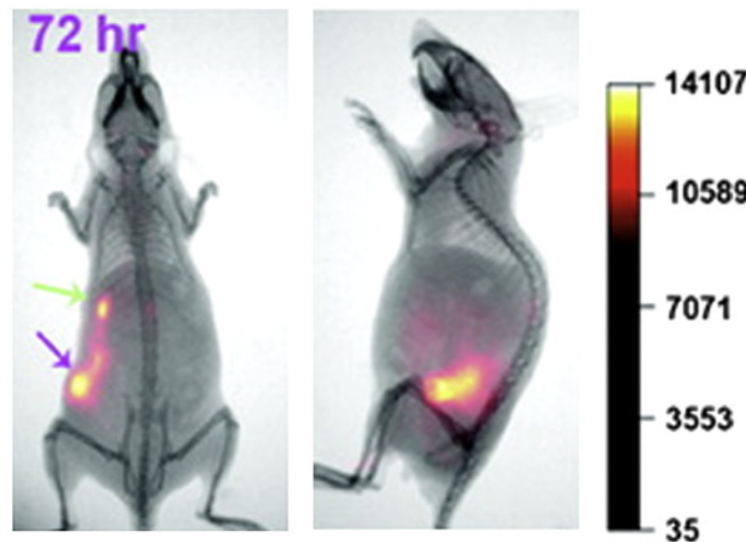

C
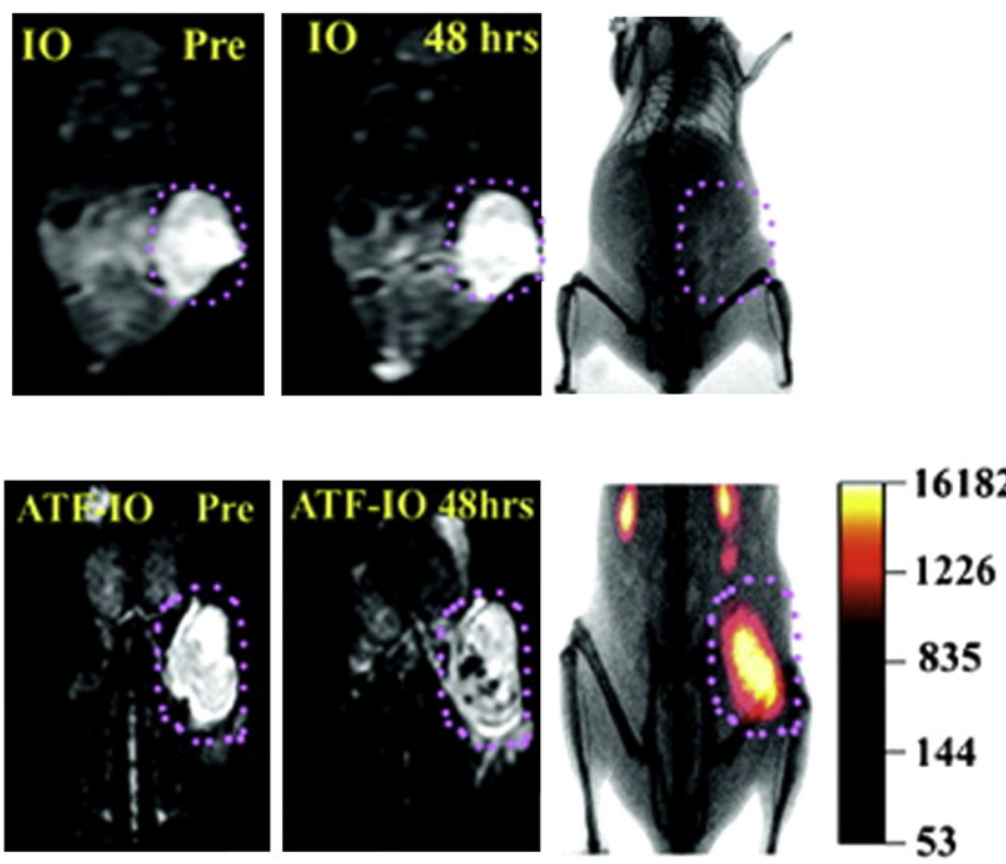

Figure 6 - Dual modality imaging of mouse mammary tumor using magnetofluorescent uPA receptor-targeted nanoagent. A) NIR optical imaging of tumor targeting and tissue distribution of targeted nanoparticles over time. Pink arrows, tumor; green arrows, kidneys. B) Back and side images are at $72 \mathrm{~h}$. C) Simultaneous magnetic resonance and optical imaging of mammary tumor. Optical imaging reveals the NIR signal in the tumor area corresponding well with the MR imaging results (bottom, pink dashed lined). $T_{2}$ contrast change is not detected in the s.c. tumor of the mouse that received non-targeted iron oxide nanoparticles (top). Reproduced with permission from Yang et al. (2009b). 
The luteinizing hormone releasing hormone (LHRH) receptor is expressed in over $50 \%$ of breast cancer cells, thus LHRH bearing nanoparticles become a viable platform for the imaging of tumors. Recent work by Meng et al. (2009) details the synthesis of iron oxide nanoparticles coated with LHRH, which were subsequently tested in vitro and in vivo in the relevant models. Studies confirmed that the targeted nanoparticles were able to serve as target-specific contrast agents, providing sub-millimeter spatial resolution for the detection of breast cancer. Moreover, the authors hypothesized that the cellular localization of LHRH-targeted nanoparticles may also be utilized for treatment purposes by loading the particles with therapeutic agents.
4.2. Targeted nanomaterials for the detection of prostate cancer

Prostate cancer is the most common cancer in men, and the second most deadly (American Cancer Society, 2009). As a result of slow tumor growth, the disease is usually not detected early on, which results in the spreading of the disease via metastasis in $30-50 \%$ patients diagnosed with prostate cancer (Stephenson and Eastham, 2005; Stephenson et al., 2006; Landis et al., 1999). Thus, improved techniques are urgently needed in order to detect the disease in its early stages to improve the survival rates of patients.
White light reflectance

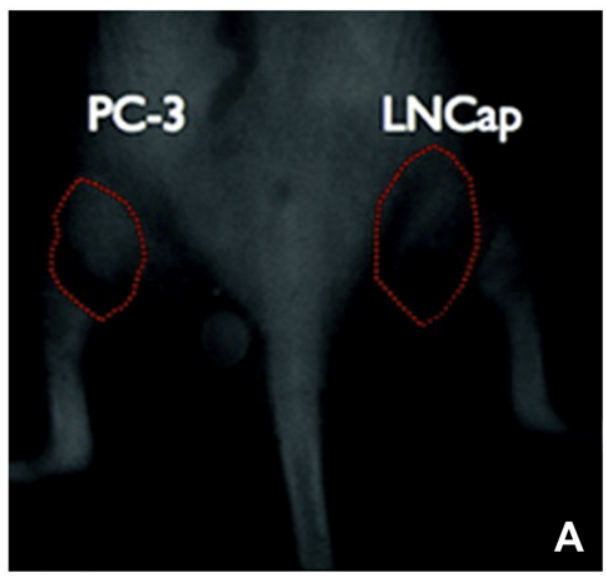

A
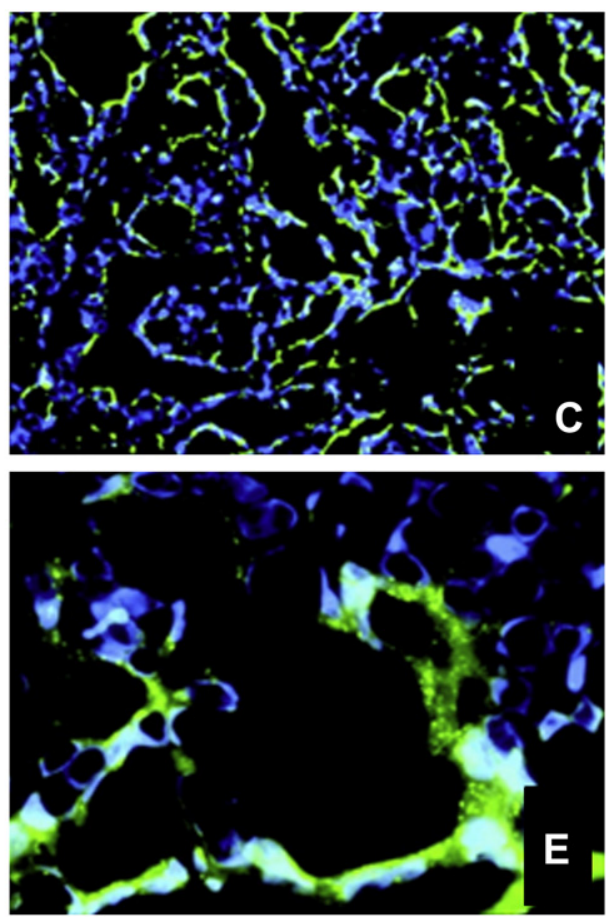

\section{FMT}

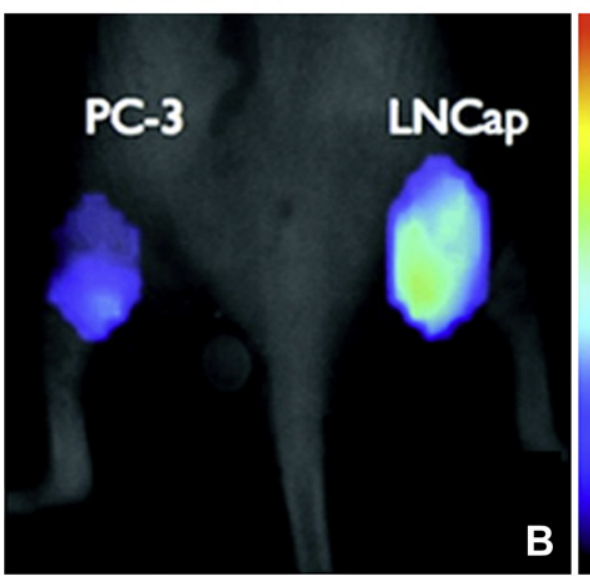

$\mathrm{nmol} / \mathrm{L}$

56

43

31

18

6
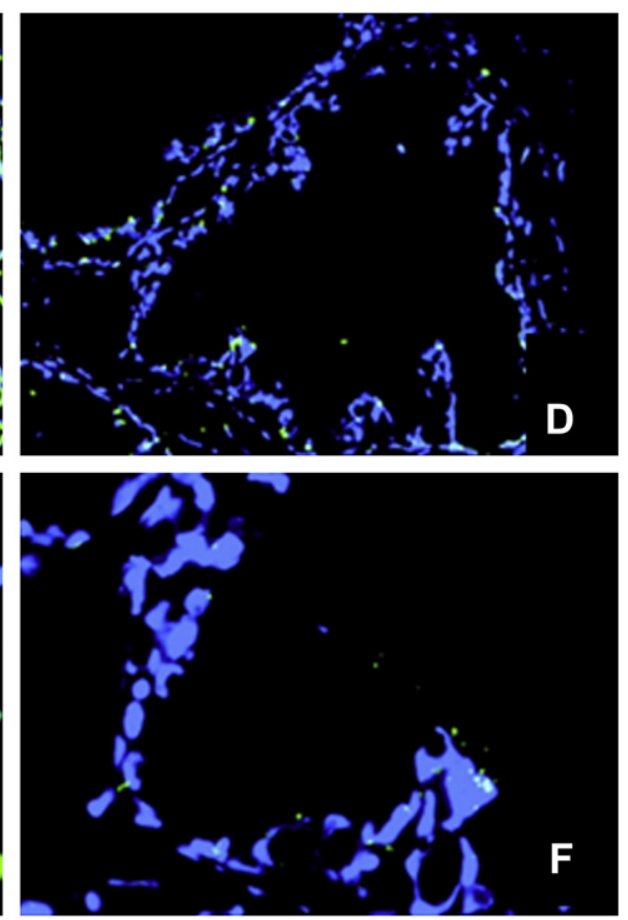

Figure 7 - Imaging of prostate cancer. A and B) Mice bearing tumors derived from PC-3 (left flank) or LNCaP (right flank) were coinjected with IPL-modified nanoagent and then imaged, illustrating preferential localization by HPN-expressing cancers. The HPN-targeted nanoparticles were also able to identify human prostate cancer. The IPL-modified nanoparticle was incubated with tissue microarrays consisting of six normal and five cancer human radical prostatectomy specimens. Results shown are representative images. $\mathrm{C}$ and $\mathrm{E}$ ) binding of nanoagent to cancer glands. D and F) binding to benign glands. Reproduced with permission from Kelly et al. (2008). 
Kelly et al. (2008) have reported the discovery of a novel peptide sequence (IPL) specific for hepsin (HPN), a type II transmembrane serine protease that is expressed only in prostate cancer cells, not in the healthy prostate, via phage screening. In order to utilize this peptide to image prostate cancer, the authors conjugated it to amine modified magnetofluorescent nanoparticles. In the first step of the synthesis, the free amine groups on the particle surface were activated toward covalent conjugation to a free thiol contained at the C-terminus of the peptide. Fluorescein labeled IPL was anchored to nanoparticles yielding approximately 11 peptides per particle. IPL decorated particles were then tested in vivo in HPN-expressing LNCaP xenografts as well as non-HPNexpressing PC3 xenografts. The targeted nanoparticles demonstrated high selectivity for the HPN-expressing human prostate cancer cells, which makes them potential candidates for early detection of prostate cancer (Figure 7).

Gao et al. (2004) have published interesting results regarding the use of antibody-targeted QDs for the in vivo imaging of prostate cancer. CdSe/ZnS QDs were initially coated with trin-octylphosphine oxide (TOPO) and an amphiphilic polymer to prevent QD hydrolysis and enzymatic degradation in vivo. The hydrophilic surface of the polymer, with 400-500 carboxylic acid groups, offers the ability to conjugate a wide variety of targeting molecules, as well as therapeutic agents on the QD surface. Initially, PEG molecules were conjugated to the polymer-coated QDs in order to increase the effective blood half-lives of the agents. Subsequently, prostate specific membrane antigen (PSMA) MAb was anchored to the free carboxylic acids of the QDs. Antibody conjugated QDs were evaluated in PSMA expressing C4-2 human prostate cancer cell lines. In vitro studies showed that the antibody bearing QDs bound with high affinity to PSMA-positive C4-2 cells, while the QD-PEG (QDs without PSMA antibody) conjugates exhibited minimal binding to the cells, most likely via nonspecific interactions. For in vivo imaging studies, the QDPSMA antibody and QD-PEG conjugates were injected into tumor bearing nude mice. While QD-PSMA antibody conjugate readily localized to tumor sites as confirmed by a strong fluorescence signal, non-targeted QD-PEG conjugate showed minimal uptake, most likely through passive targeting. Histological analysis of QD uptake and distribution studies further illustrated that the QD-PSMA antibody conjugates specifically targeted PSMA-positive human prostate tumor sites.

Lastly, Farokhzad and coworkers have engineered QD-based "smart" multifunctional aptamer targeted nanoparticles that are capable of delivering and sensing the release of the chemotherapeutic doxorubicin (Dox) (Bagalkot et al., 2007). Briefly, CdSe/ZnS core shell QDs were decorated with amineterminated A10 RNA aptamer by employing carbodiimide activation and coupling chemistry. The A10 RNA aptamers specifically binds to PSMA expressing LNCaP human prostate cancer cells. When it is in double stranded form, the stem region acts as a host for Dox via non-covalent interactions. The main idea behind the "smart" multifunctional nanoparticles is that it consists of bi-FRET system, QD-Dox and Dox-aptamer are donor-acceptor pairs. When Dox was introduced to QD-Apt nanoparticles, the fluorescence of the QD was essentially turned "off," as it was quenched by the Dox absorbance. At the same time, Dox fluorescence was quenched by the RNA aptamer resulting in the fluorescence "off" bi-FRET system. When nanoparticles bind to PSMA and are internalized, Dox is released and the system consequently regains its ability to fluoresce. This "smart" system has been tested in vitro using LNCaP positive and PSMA-negative PC3 human prostate cancer cell lines by incubating the cells with QD-Apt(Dox) nanoparticles. In vitro studies demonstrated that while very faint fluorescence was observed at $0 \mathrm{~h}$ of incubation of QD-Apt (Dox) with LNCaP cells, when incubated for $1.5 \mathrm{~h}$ further the fluorescence signal turned "on" confirming the delivery of Dox to the target cells. It was also noted that QD-Apt nanoparticles did not show inherent cytotoxicity to the LNCaP and PC3 cells. Thus, cytotoxicity of QD-Apt(Dox) nanoparticles was associated with the release of Dox to the cells.

\section{Conclusions and outlook}

One of the greatest advantages of nanotechnology is the ability to generate materials functionalized with a wide variety of ligands. This versatility allows for the creation of agents with specially tailored properties, including prolonged blood halflives, and the potential to target sites of interest. With regard to the detection of cancers, this has led to the description of a seemingly infinite array of particles, each able to localize to different biological targets, and bearing a multitude of imaging components. Although not described in detail here, one of the greatest advantages of these nanoagents is their ability to co-deliver therapeutic moieties. These theranostic (therapeutic and diagnostic) nanoparticles allow for the determination of the localization, release, and therapeutic efficacy of the multifunctional nanoagent, enabling the enhanced diagnosis and treatment of cancers. While these agents seem to offer several advantages over conventional therapeutics, there are several questions that arise. Is it necessary to administer imaging agents each time a patient needs therapy? Can the mismatch between the dose required for imaging and therapy be reconciled? Can such complex agents clear regulatory hurdles? Given the rapid pace of progress in the field of nanomedicine, these and other questions may be answered sooner than anticipated.

\section{Acknowledgments}

This work was supported in part by NIH grants R21HL093607 (JM) and U01-HL080731 (JM, RW)

\section{R E F E R E N C E S}

Aime, S., Castelli, D.D., Crich, S.G., Gianolio, E., Terreno, E., 2009. Pushing the sensitivity envelope of lanthanide-based magnetic resonance imaging (MRI) contrast agents for molecular imaging applications. Acc. Chem. Res. 42, 822-831.

American Cancer Society, 2009. Cancer Facts \& Figures 2009. American Cancer Society, Atlanta.

Bagalkot, V., Zhang, L., Levy-Nissenbaum, E., Jon, S., Kantoff, P.W., Langer, R., Farokhzad, O.C., 2007. Quantum dot-aptamer conjugates for synchronous cancer imaging, 
therapy, and sensing of drug delivery based on bi-fluorescence resonance energy transfer. Nano. Lett. 7, 3065-3070.

Biju, V., Mundayoor, S., Omkumar, R.V., Anas, A., Ishikawa, M., 2010. Bioconjugated quantum dots for cancer research: present status, prospects and remaining issues. Biotechnol. Adv. 28, 199-213.

Cairns, R.A., Khokha, R., Hill, R.P., 2003. Molecular mechanisms of tumor invasion and metastasis: an integrated view. Curr. Mol. Med. 3, 659-671.

Caravan, P., Ellison, J.J., McMurry, T.J., Lauffer, R.B., 1999. Gadolinium(III) chelates as MRI contrast agents: structure, dynamics, and applications. Chem. Rev. 99, 2293-2352.

Chen, T.J., Cheng, T.H., Chen, C.Y., Hsu, S.C., Cheng, T.L., Liu, G.C., Wang, Y.M., 2009. Targeted herceptin-dextran iron oxide nanoparticles for noninvasive imaging of HER2/neu receptors using MRI. J. Biol. Inorg. Chem. 14, 253-260.

Cheng, Z., Wu, Y., Xiong, Z., Gambhir, S.S., Chen, X., 2005. Nearinfrared fluorescent RGD peptides for optical imaging of integrin alphavbeta3 expression in living mice. Bioconjug. Chem. 16, 1433-1441.

Christiansen, J.P., Lindner, J.R., 2005. Molecular and cellular imaging with targeted contrast ultrasound. Proc. IEEE 93, 809-818.

Couturier, O., Luxen, A., Chatal, J.F., Vuillez, J.P., Rigo, P., Hustinx, R., 2004. Fluorinated tracers for imaging cancer with positron emission tomography. Eur. J. Nucl. Med. Mol. Imaging 31, 1182-1206.

Curati, W.L., Halevy, A., Gibson, R.N., Carr, D.H., Blumgart, L.H., Steiner, R.E., 1988. Ultrasound, CT, and MRI comparison in primary and secondary tumors of the liver. Gastrointest. Radiol. 13, 123-128.

Dancey, G., Begent, R.H., Meyer, T., 2009. Imaging in targeted delivery of therapy to cancer. Target. Oncol. 4, 201-217.

Dayton, P.A., Zhao, S., Bloch, S.H., Schumann, P., Penrose, K., Matsunaga, T.O., Zutshi, R., Doinikov, A., Ferrara, K.W., 2006. Application of ultrasound to selectively localize nanodroplets for targeted imaging and therapy. Mol. Imaging 5, 160-174.

Devaraj, N.K., Keliher, E.J., Thurber, G.M., Nahrendorf, M., Weissleder, R., 2009. ${ }^{18} \mathrm{~F}$ labeled nanoparticles for in vivo PETCT imaging. Bioconjug. Chem. 20, 397-401.

Ellington, A.D., Szostak, J.W., 1990. In vitro selection of RNA molecules that bind specific ligands. Nature 346, 818-822.

Enochs, W.S., Harsh, G., Hochberg, F., Weissleder, R., 1999. Improved delineation of human brain tumors on MR images using a long-circulating, superparamagnetic iron oxide agent. J. Magn. Reson. Imaging 9, 228-232.

Farokhzad, O.C., Karp, J.M., Langer, R., 2006. Nanoparticle-aptamer bioconjugates for cancer targeting. Expert Opin. Drug Deliv. 3, 311-324.

Fass, L., 2008. Imaging and cancer: a review. Mol. Oncol. 2, 115-152.

Felding-Habermann, B., 2003. Integrin adhesion receptors in tumor metastasis. Clin. Exp. Metastasis 20, 203-213.

Ferrari, M., 2005. Cancer nanotechnology: opportunities and challenges. Nat. Rev. Cancer 5, 161-171.

Fisher, J.L., Field, C.L., Zhou, H., Harris, T.L., Henderson, M.A., Choong, P.F., 2000. Urokinase plasminogen activator system gene expression is increased in human breast carcinoma and its bone metastases-a comparison of normal breast tissue, non-invasive and invasive carcinoma and osseous metastases. Breast Cancer Res. Treat. 61, 1-12.

Gao, X., Cui, Y., Levenson, R.M., Chung, L.W., Nie, S., 2004. In vivo cancer targeting and imaging with semiconductor quantum dots. Nat. Biotechnol. 22, 969-976.

Graves, E.E., Weissleder, R., Ntziachristos, V., 2004. Fluorescence molecular imaging of small animal tumor models. Curr. Mol. Med. 4, 419-430.

Guo, K.T., Paul, A., Schichor, C., Ziemer, G., Wendel, H.P., 2008. CELL-SELEX: novel perspectives of aptamer-based therapeutics. Int. J. Mol. Sci. 9, 668-678.
Hamoudeh, M., Kamleh, M.A., Diab, R., Fessi, H., 2008. Radionuclides delivery systems for nuclear imaging and radiotherapy of cancer. Adv. Drug Deliv. Rev. 60, 1329-1346.

Han, B., Nakamura, M., Mori, I., Nakamura, Y., Kakudo, K., 2005. Urokinase-type plasminogen activator system and breast cancer (Review). Oncol. Rep. 14, 105-112.

Harisinghani, M.G., Weissleder, R., 2004. Sensitive, noninvasive detection of lymph node metastases. PLoS Med. 1, e66.

Harisinghani, M.G., Saini, S., Weissleder, R., Rubin, D., deLange, E., Harms, S., Weinreb, J., Small, W., Sukerkar, A., Brown, J.J., Zelch, J., Lucas, M., Morris, M., Hahn, P.F., 2001. Splenic imaging with ultrasmall superparamagnetic iron oxide ferumoxtran-10 (AMI-7227): preliminary observations. J. Comput. Assist. Tomogr. 25, 770-776.

Harisinghani, M.G., Barentsz, J., Hahn, P.F., Deserno, W.M., Tabatabaei, S., van de Kaa, C.H., de la Rosette, J., Weissleder, R., 2003. Noninvasive detection of clinically occult lymph-node metastases in prostate cancer. N. Engl. J. Med. 348, 2491-2499.

Harrington, K.J., Mohammadtaghi, S., Uster, P.S., Glass, D., Peters, A.M., Vile, R.G., Stewart, J.S., 2001. Effective targeting of solid tumors in patients with locally advanced cancers by radiolabeled pegylated liposomes. Clin. Cancer Res. 7, 243-254.

Herbst, R.S., 2004. Review of epidermal growth factor receptor biology. Int. J. Radiat. Oncol. Biol. Phys. 59, 21-26.

Hilderbrand, S.A., Weissleder, R., 2010. Near-infrared fluorescence: application to in vivo molecular imaging. Curr. Opin. Chem. Biol. 14, 71-79.

Hoffman, R.M., 2009. Imaging cancer dynamics in vivo at the tumor and cellular level with fluorescent proteins. Clin. Exp. Metastasis 26, 345-355.

Hornak, J.P., 2010. The basics of MRI. http://www.cis.rit.edu/ htbooks/mri/.

Hung, M.C., Lau, Y.K., 1999. Basic science of HER-2/neu: a review. Semin. Oncol. 26, 51-59.

Iyer, A.K., Khaled, G., Fang, J., Maeda, H., 2006. Exploiting the enhanced permeability and retention effect for tumor targeting. Drug Discov. Today 11, 812-818.

Jensen, J.A., 2007. Medical ultrasound imaging. Prog. Biophys. Mol. Biol. 93, 153-165.

Jiang, S., Gnanasammandhan, M.K., Zhang, Y., 2010. Optical imaging-guided cancer therapy with fluorescent nanoparticles. J. R Soc. Interface 7, 3-18.

Jobsis, F.F., 1977. Noninvasive, infrared monitoring of cerebral and myocardial oxygen sufficiency and circulatory parameters. Science 198, 1264-1267.

Kaneda, M.M., Caruthers, S., Lanza, G.M., Wickline, S.A., 2009. Perfluorocarbon nanoemulsions for quantitative molecular imaging and targeted therapeutics. Ann. Biomed. Eng. 37, 1922-1933.

Kaufmann, B.A., Lindner, J.R., 2007. Molecular imaging with targeted contrast ultrasound. Curr. Opin. Biotechnol. 18, $11-16$

Kelly, K.A., Setlur, S.R., Ross, R., Anbazhagan, R., Waterman, P., Rubin, M.A., Weissleder, R., 2008. Detection of early prostate cancer using a hepsin-targeted imaging agent. Cancer Res. 68, 2286-2291.

Kherlopian, A.R., Song, T., Duan, Q., Neimark, M.A., Po, M.J., Gohagan, J.K., Laine, A.F., 2008. A review of imaging techniques for systems biology. BMC Syst. Biol. 2, 74.

Landis, S.H., Murray, T., Bolden, S., Wingo, P.A., 1999. Cancer statistics, 1999. CA Cancer J. Clin. 49, 8-31. 1.

Lanza, G.M., Winter, P.M., Caruthers, S.D., Hughes, M.S., Cyrus, T., Marsh, J.N., Neubauer, A.M., Partlow, K.C., Wickline, S.A., 2006. Nanomedicine opportunities for cardiovascular disease with perfluorocarbon nanoparticles. Nanomedicine 1, 321-329. 
Lee, H.Y., Li, Z., Chen, K., Hsu, A.R., Xu, C., Xie, J., Sun, S., Chen, X., 2008. PET/MRI dual-modality tumor imaging using arginineglycine-aspartic (RGD)-conjugated radiolabeled iron oxide nanoparticles. J. Nucl. Med. 49, 1371-1379.

Levy-Nissenbaum, E., Radovic-Moreno, A.F., Wang, A.Z., Langer, R., Farokhzad, O.C., 2008. Nanotechnology and aptamers: applications in drug delivery. Trends Biotechnol. 26, $442-449$.

Li, X., Du, X., Huo, T., Liu, X., Zhang, S., Yuan, F., 2009. Specific targeting of breast tumor by octreotide-conjugated ultrasmall superparamagnetic iron oxide particles using a clinical 3.0Tesla magnetic resonance scanner. Acta Radiol. 50, 583-594.

Lin, M.M., Kim do, K., El Haj, A.J., Dobson, J., 2008. Development of superparamagnetic iron oxide nanoparticles (SPIONS) for translation to clinical applications. IEEE Trans. Nanobioscience 7, 298-305.

Lin, W., Hyeon, T., Lanza, G.M., Zhang, M., Meade, T.J., 2009. Magnetic nanoparticles for early detection of cancer by magnetic resonance imaging. MRS Bull. 34, 441-448.

Low, P.S., Kularatne, S.A., 2009. Folate-targeted therapeutic and imaging agents for cancer. Curr. Opin. Chem. Biol. 13, 256-262.

Maeda, H., 2001. The enhanced permeability and retention (EPR) effect in tumor vasculature: the key role of tumor-selective macromolecular drug targeting. Adv. Enzyme Regul. 41, 189-207.

Maeda, H., Wu, J., Sawa, T., Matsumura, Y., Hori, K., 2000. Tumor vascular permeability and the EPR effect in macromolecular therapeutics: a review. J. Control Release 65, 271-284.

Mardirossian, G., Brill, A.B., Dwyer, K.M., Kahn, D., Nelp, W., 1996. Radiation absorbed dose from indium-111-CYT-356. J. Nucl. Med. 37, 1583-1588.

Matsumura, Y., Maeda, H., 1986. A new concept for macromolecular therapeutics in cancer chemotherapy: mechanism of tumoritropic accumulation of proteins and the antitumor agent smancs. Cancer Res. 46, 6387-6392.

Maurer, A.H., 2008. Combined imaging modalities: PET/CT and SPECT/CT. Health Phys. 95, 571-576.

McCarthy, J.R., Weissleder, R., 2008. Multifunctional magnetic nanoparticles for targeted imaging and therapy. Adv. Drug Deliv. Rev. 60, 1241-1251.

McCarthy, J.R., Kelly, K.A., Sun, E.Y., Weissleder, R., 2007. Targeted delivery of multifunctional magnetic nanoparticles. Nanomedicine (Lond) 2, 153-167.

Meng, J., Fan, J., Galina, G., Branca, R.T., Clasen, P.L., Ma, S. Zhou, J., Leuschner, C., Kumar, C.S.R.R., Hormes, J., Otiti, T., Beye, A.C., Harmer, M.P., Kiely, C.J., Warren, W., Haataja, M.P., Soboyejo, W.O., 2009. LHRH-functionalized supermagnetic iron oxide nanoparticles for breast cancer targeting and contrast enhancement in MRI. Mater. Sci. Eng. C 29, 1467-1479.

Mittelstaedt, C.A., 1980. Ultrasound as a useful imaging modality for tumor detection and staging. Cancer Res. 40, 3072-3078.

Montet, X., Weissleder, R., Josephson, L., 2006. Imaging pancreatic cancer with a peptide-nanoparticle conjugate targeted to normal pancreas. Bioconjug. Chem. 17, 905-911.

Moore, G.E., Peyton, W.T., et al., 1948. The clinical use of fluorescein in neurosurgery; the localization of brain tumors. J. Neurosurg. 5, 392-398.

Mulder, W.J., Strijkers, G.J., Habets, J.W., Bleeker, E.J., van der Schaft, D.W., Storm, G., Koning, G.A., Griffioen, A.W., Nicolay, K., 2005. MR molecular imaging and fluorescence microscopy for identification of activated tumor endothelium using a bimodal lipidic nanoparticle. FASEB J. 19, 2008-2010.

Nahrendorf, M., Waterman, P., Thurber, G., Groves, K., Rajopadhye, M., Panizzi, P., Marinelli, B., Aikawa, E. Pittet, M.J., Swirski, F.K., Weissleder, R., 2009. Hybrid in vivo
FMT-CT imaging of protease activity in atherosclerosis with customized nanosensors. Arterioscler. Thromb. Vasc. Biol. 29, 1444-1451.

Ntziachristos, V., 2006. Fluorescence molecular imaging. Annu. Rev. Biomed. Eng. 8, 1-33.

Park, J.H., von Maltzahn, G., Ruoslahti, E., Bhatia, S.N., Sailor, M.J. 2008. Micellar hybrid nanoparticles for simultaneous magnetofluorescent imaging and drug delivery. Angew. Chem. Int. Ed. Engl. 47, 7284-7288.

Phillips, W.T., 1999. Delivery of gamma-imaging agents by liposomes. Adv. Drug Deliv. Rev. 37, 13-32.

Rapoport, N., Gao, Z., Kennedy, A., 2007. Multifunctional nanoparticles for combining ultrasonic tumor imaging and targeted chemotherapy. J. Natl. Cancer Inst. 99, 1095-1106.

Retel, V.P., Hummel, M.J., van Harten, W.H., 2009. Review on early technology assessments of nanotechnologies in oncology. Mol. Oncol. 3, 394-401.

Saini, S., Stark, D.D., Hahn, P.F., Bousquet, J.C., Introcasso, J., Wittenberg, J., Brady, T.J., Ferrucci, J.T.J., 1987. Ferrite particles: a superparamagnetic MR contrast agent for enhanced detection of liver carcinoma. Radiology 162, 217-222.

Saini, S., Sharma, R., Baron, R.L., Turner, D.A., Ros, P.R., Hahn, P.F., Small, W.C., Delange, E.E., Stillman, A.E., Edelman, R.R., Runge, V.M., Outwater, E.K., 2000. Multicentre dose-ranging study on the efficacy of USPIO ferumoxtran-10 for liver MR imaging. Clin. Radiol. 55, 690-695.

Saltz, L., Easley, C., Kirkpatrick, P., 2006. Panitumumab. Nat. Rev. Drug Discov. 5, 987-988.

Sampath, L., Kwon, S., Ke, S., Wang, W., Schiff, R., Mawad, M.E., Sevick-Muraca, E.M., 2007. Dual-labeled trastuzumab-based imaging agent for the detection of human epidermal growth factor receptor 2 overexpression in breast cancer. J. Nucl. Med. $48,1501-1510$

Santra, S., Dutta, D., Walter, G.A., Moudgil, B.M., 2005. Fluorescent nanoparticle probes for cancer imaging. Technol. Cancer Res. Treat. 4, 593-602.

Simberg, D., Duza, T., Park, J.H., Essler, M., Pilch, J., Zhang, L., Derfus, A.M., Yang, M., Hoffman, R.M., Bhatia, S., Sailor, M.J., Ruoslahti, E., 2007. Biomimetic amplification of nanoparticle homing to tumors. Proc. Natl. Acad. Sci. U.S.A 104, 932-936.

Stark, D.D., Moseley, M.E., Bacon, B.R., Moss, A.A., Goldberg, H.I., Bass, N.M., James, T.L., 1985. Magnetic resonance imaging and spectroscopy of hepatic iron overload. Radiology 154, 137-142.

Stephenson, A.J., Eastham, J.A., 2005. Role of salvage radical prostatectomy for recurrent prostate cancer after radiation therapy. J. Clin. Oncol. 23, 8198-8203.

Stephenson, A.J., Scardino, P.T., Eastham, J.A., Bianco, F.J.J., Dotan, Z.A., Fearn, P.A., Kattan, M.W., 2006. Preoperative nomogram predicting the 10 -year probability of prostate cancer recurrence after radical prostatectomy. J. Natl. Cancer Inst. 98, 715-717.

Tang, Y., Kim, M., Carrasco, D., Kung, A.L., Chin, L., Weissleder, R., 2005. In vivo assessment of RAS-dependent maintenance of tumor angiogenesis by real-time magnetic resonance imaging. Cancer Res. 65, 8324-8330.

Tassa, C., Duffner, J.L., Lewis, T.A., Weissleder, R., Schreiber, S.L., Koehler, A.N., Shaw, S.Y., 2010. Binding affinity and kinetic analysis of targeted small molecule-modified nanoparticles. Bioconjug. Chem. 21, 14-19.

Ting, G., Chang, C.H., Wang, H.E., 2009. Cancer nanotargeted radiopharmaceuticals for tumor imaging and therapy. Anticancer Res. 29, 4107-4118.

Tran, T., Engfeldt, T., Orlova, A., Sandstrom, M., Feldwisch, J., Abrahmsen, L., Wennborg, A., Tolmachev, V., Karlstrom, A.E., 2007a. (99m)Tc-maEEE-Z(HER2:342), an Affibody moleculebased tracer for the detection of HER2 expression in malignant tumors. Bioconjug. Chem. 18, 1956-1964. 
Tran, T.D., Caruthers, S.D., Hughes, M., Marsh, J.N., Cyrus, T., Winter, P.M., Neubauer, A.M., Wickline, S.A., Lanza, G.M., 2007b. Clinical applications of perfluorocarbon nanoparticles for molecular imaging and targeted therapeutics. Int. J. Nanomedicine 2, 515-526.

Tuerk, C., Gold, L., 1990. Systematic evolution of ligands by exponential enrichment: RNA ligands to bacteriophage T4 DNA polymerase. Science 249, 505-510.

Wang, K., Wang, K., Li, W., Huang, T., Li, R., Wang, D., Shen, B., Chen, X., 2009. Characterizing breast cancer xenograft epidermal growth factor receptor expression by using nearinfrared optical imaging. Acta Radiol. 50, 1095-1103.

Warburg, O., 1956. On the origin of cancer cells. Science 123, 309-314.

Weissleder, R., Hahn, P.F., Stark, D.D., Rummeny, E., Saini, S., Wittenberg, J., Ferrucci, J.T., 1987a. MR imaging of splenic metastases: ferrite-enhanced detection in rats. AJR Am. J. Roentgenol. 149, 723-726.

Weissleder, R., Stark, D.D., Compton, C.C., Wittenberg, J., Ferrucci, J.T., 1987b. Ferrite-enhanced MR imaging of hepatic lymphoma: an experimental study in rats. AJR Am. J. Roentgenol 149, 1161-1165.

Weissleder, R., Kelly, K., Sun, E.Y., Shtatland, T., Josephson, L., 2005. Cell-specific targeting of nanoparticles by multivalent attachment of small molecules. Nat. Biotechnol. 23, 1418-1423.

Xing, Y., Rao, J., 2008. Quantum dot bioconjugates for in vitro diagnostics \& in vivo imaging. Cancer Biomark. 4, 307-319.

Yang, L., Mao, H., Wang, Y.A., Cao, Z., Peng, X., Wang, X., Duan, H., Ni, C., Yuan, Q., Adams, G., Smith, M.Q., Wood, W.C., Gao, X., Nie, S., 2009a. Single chain epidermal growth factor receptor antibody conjugated nanoparticles for in vivo tumor targeting and imaging. Small 5, 235-243.

Yang, L., Peng, X.H., Wang, Y.A., Wang, X., Cao, Z., Ni, C., Karna, P., Zhang, X., Wood, W.C., Gao, X., Nie, S., Mao, H., 2009b. Receptor-targeted nanoparticles for in vivo imaging of breast cancer. Clin. Cancer Res. 15, 4722-4732.

Yuan, F., Dellian, M., Fukumura, D., Leunig, M., Berk, D.A., Torchilin, V.P., Jain, R.K., 1995. Vascular permeability in a human tumor xenograft: molecular size dependence and cutoff size. Cancer Res. 55, 3752-3756.

Zhang, D., Feng, X.Y., Henning, T.D., Wen, L., Lu, W.Y., Pan, H., $\mathrm{Wu}, \mathrm{X}$., Zou, L.G., 2009. MR imaging of tumor angiogenesis using sterically stabilized Gd-DTPA liposomes targeted to CD105. Eur. J. Radiol. 70, 180-189.

Zhang, H., Ma, Y., Sun, X.L., 2010. Recent developments in carbohydrate-decorated targeted drug/gene delivery. Med. Res. Rev. 30, 270-289. 